\title{
A Compactification of the Universal Moduli Space of Principal G-Bundles
}

\author{
Ángel Luis Muñoz Castañeda®
}

\begin{abstract}
Let $G$ be a semisimple linear algebraic group, $\rho: G \hookrightarrow \operatorname{SL}(V)$ a finite-dimensional faithful representation, $g \geq 2$ a natural number, and $\delta$ a positive rational number. We prove the existence of a compactification of the universal moduli space of semistable principal $G$-bundles over $\overline{\mathrm{M}}_{g}$, provided that $\delta$ is sufficiently large, having the following property: the fibers over singular curves are the moduli spaces of $\delta$-semistable singular principal $G$-bundles.
\end{abstract}

Mathematics Subject Classification. Primary 14H60; Secondary 14D20.

Keywords. Principal bundles, Universal moduli, Stable curves.

\section{Introduction}

Let $X$ be a smooth projective curve of genus $g \geq 2$ over an algebraically closed field of characteristic 0 , and $G$ a connected reductive algebraic group. In $[18,19]$, Ramanathan proved the existence of a projective moduli space, $\mathrm{M}_{X}^{(s) s}(G)$, of (semi)stable principal $G$-bundles on $X$ of a given topological type. When $G=\mathrm{GL}_{r}$, this moduli space is isomorphic to the classical moduli space of (semi)stable vector bundles constructed by Mumford and Seshadri $[15,27]$.

A problem that arises naturally is to consider a degeneration of $X$ along a discrete valuation ring, whose closed fiber is a stable curve of genus $g$, and describe the limit of a semistable principal $G$-bundle on $X$ when we approach the closed fiber. When $G=\mathrm{GL}_{r}$ (respectively, $\mathrm{Sp}_{r}, \mathrm{O}_{r}$ ), this problem is equivalent to the problem of finding the limit of a locally free sheaf of rank $r$ (respectively, together with a bilinear form satisfying certain conditions). It is well known that the solution is a torsion-free sheaf of the same rank (respectively, together with a bilinear form satisfying certain conditions) [5]. This degeneration problem has been studied by Sun when $G=\mathrm{SL}_{r}[29,30]$.

Partially supported by the Spanish MICINN under Grant no. PGC2018-099599-B-I00. 
One can also study how the moduli space of semistable principal $G$ bundles varies as the base curve varies in the moduli space of stable curves, $\overline{\mathrm{M}}_{g}$. This leads to the problem of compactifying the universal moduli space [4] of principal $G$-bundles over $\overline{\mathrm{M}}_{g}$. Regarding this problem, not much is known, except when $G=\mathrm{GL}_{r}$. In this case, Pandharipande has proved the existence of a projective moduli space and a morphism onto $\overline{\mathrm{M}}_{g}$ whose fiber over a stable curve $X$ is the moduli space of semistable torsion-free sheaves of a given rank and degree divided by $\operatorname{Aut}(X)$ (see $[3,17]$ for the particular case of $r=1)$.

For a general group $G$, Balaji has carried out one-parameter flat degenerations of the moduli space when the closed fiber of the relative curve is irreducible [1]. This is a first step in understanding how to construct a flat compactification of the universal moduli space of bundles. Concerning this problem in case $G=\mathrm{GL}(n)$, it is worth mentioning the work of Schmitt [22]. Here, a compactification of the universal moduli space is carried out by looking at vector bundles on semistable models of stable curves. It is important to note that Schmitt's construction rests on the space built by Pandharipande [17].

An alternative construction of the moduli space of principal $G$-bundles was given by Schmitt for a semisimple linear algebraic group $G$. Given a faithful representation $\rho: G \hookrightarrow \mathrm{SL}(V)$ of dimension $r$, a singular principal $G$-bundle is a pair $(\mathscr{E}, \tau)$ consisting of a locally free sheaf $\mathscr{E}$ of rank $r$ and degree 0 , and a non-trivial morphism of algebras $\tau: S^{\bullet}(V \otimes \mathscr{E})^{G} \rightarrow \mathscr{O}_{X}$. Giving $\tau$ is the same as giving a morphism $X \rightarrow \underline{\operatorname{Hom}}_{\mathscr{O}_{X}}\left(V \otimes \mathscr{O}_{X}, \mathscr{E}^{\vee}\right) / / G$, and the singular principal $G$-bundle $(\mathscr{E}, \tau)$ is said to be honest if $\operatorname{Im}(\tau)$ lies inside the subscheme $\underline{\operatorname{Isom}}_{\mathscr{O}_{X}}\left(V \otimes \mathscr{O}_{X}, \mathscr{E}^{\vee}\right) / G$. Schmitt's work is based on the following result (a particular case of [26, Proposition 9]):

$$
\left\{\begin{array}{l}
\text { isomorphism classes } \\
\text { of principal } G \text {-bundles } \\
\text { on } X
\end{array}\right\} \simeq\left\{\begin{array}{l}
\text { isomorphism classes of pairs }(\mathscr{E}, \tau) \\
\mathscr{E} \text { being a locally free sheaf of rank } \\
r \text { with trivial determinant and } \\
\tau: X \rightarrow \underline{\operatorname{Isom}}_{\mathscr{O}_{X}}\left(V \otimes \mathscr{O}_{X}, \mathscr{E}\right) / G \\
\text { a section }
\end{array}\right\} .
$$

For a given representation $\rho: G \hookrightarrow \mathrm{SL}(V)$ and a rational number $\delta \in \mathbb{Q}_{>0}$, it is proved in [23] the existence of a projective moduli space, $\operatorname{SPB}(\rho)_{X, P}^{\delta-(s) s}$, of $\delta$-(semi)stable singular principal $G$-bundles with Hilbert polynomial $P$ over a smooth projective curve $X$. Furthermore, for $\delta \gg 0$ and $P(k)=r k+r(1-g)$, it is also proved that every $\delta$-semistable singular principal $G$-bundle is honest and that $\operatorname{SPB}(\rho)_{X, P}^{\delta-(s) s}$ is isomorphic to $\mathrm{M}_{X}^{(s) s}(G)$. It is worth pointing out that it is possible to prove the existence of these moduli spaces when working over more general base schemes $[2,11,14]$. Considering everything said so far, Schmitt's approach seems suitable for tackling the problems mentioned above when the linear algebraic group $G$ is different from $\mathrm{GL}_{r}$.

This work aims to prove the existence of a compactification of the moduli problem defined by pairs $(X, \mathscr{P})$, where $X$ is a smooth projective curve of genus $g$ and $\mathscr{P}$ is a semistable principal $G$-bundle. This generalizes the 
construction for vector bundles given by Pandharipande [17]. The approach we will follow is the approach taken by Schmitt; therefore, we will substitute $\mathscr{P}$ by its corresponding singular principal $G$-bundle. A key component in the theory of singular principal bundles is the notion of swamp, which over a reduced projective curve is a pair $(\mathscr{F}, \phi)$, where $\mathscr{F}$ is a coherent sheaf of pure dimension one and $\phi:\left(\mathscr{F}^{\otimes a}\right)^{\oplus b} \rightarrow \mathscr{D}$ is a morphism of $\mathscr{O}_{X}$-modules $[11,14]$. Here, $a, b$ and $\mathscr{D}$ are fixed input data. From [14], it follows that the construction of the universal moduli space of singular principal bundles reduces, essentially, to the construction of the universal moduli space of swamps. Thus, a significant part of this work is focused on the universal moduli space of these objects.

The precise statement of the main result is as follows.

Theorem 1.1. Let $G$ be a semisimple linear algebraic group, $\rho: G \hookrightarrow \operatorname{SL}(V)$ a faithful representation of dimension $r, g \geq 2$ a natural number, and $\delta \in \mathbb{Q}_{>0}$ a rational number. There exists a projective scheme $\operatorname{SPB}(\rho)_{r, g}^{\delta-(\mathrm{s}) \mathrm{s}}$, together with a map $\Theta_{b}: \operatorname{SPB}(\rho)_{r, g}^{\delta-(\mathrm{s}) \mathrm{s}} \rightarrow \overline{\mathrm{M}}_{g}$, such that for any stable curve $[X] \in \overline{\mathrm{M}}_{g}$, $\Theta_{b}^{-1}([X])=\operatorname{SPB}(\rho)_{r, X}^{\delta-(\mathrm{s}) \mathrm{s}} / \operatorname{Aut}(X)$, where $\operatorname{SPB}(\rho)_{r, X}^{\delta-(\mathrm{s}) \mathrm{s}}$ is the moduli space of $\delta$-(semi)stable singular principal $G$-bundles, $(\mathscr{F}, \tau)$, on $X$ with $\mathscr{F}$ of uniform rank $r$ and degree 0. If $\delta$ is large enough, $\Theta_{b}^{-1}([X])=\mathrm{M}_{X}^{(s) s}(G) / \operatorname{Aut}(X)$ for every smooth curve $[X] \in \overline{\mathrm{M}}_{g}$.

The main technical result necessary to prove the previous theorem is the following.

Theorem (Theorem 3.6). Fix $g, h, C \in \mathbb{N}$ with $g \geq 2$, and a finite set $\underline{P}$ of polynomials of degree one with integral coefficients. There is a natural number $N_{0}$, depending only on $\underline{P}, C, g$ and $h$, such that for every Cohen-Macaulay projective and connected curve of genus $g$ with a very ample invertible sheaf $\mathscr{O}_{X}(1)$ of degree $h$ and every coherent sheaf of pure dimension one, $\mathscr{F}$, over $X$ with Hilbert polynomial in $\underline{P}$ satisfying $A$ coherent sheaf $\mu_{\max }(\mathscr{F}) \leq C$, the following holds: for all $k \geq N_{0}, h^{1}(X, \mathscr{F}(k))=0$, and $\mathscr{F}(k)$ is generated by its global sections.

This result is necessary to prove the existence of a relative Quot scheme over the parameter space of the universal curve of genus $g$ containing every coherent sheaf of pure dimension one with a given Hilbert polynomial that appears in a $\delta$-semistable singular principal bundle. Note that in case of $\underline{P}$ consist of one polynomial, $P(n)=\alpha n+\beta$, and $C$ be equal to $\beta / \alpha$, Theorem 3.6 implies the existence of a Quot scheme, relative to the parameter space for stable curves of genus $g$, which contains every semistable sheaf with Hilbert polynomial $P$ (this particular case was proved in [17, Section 5, Section 6]).

Following the proof of [7, Theorem 3.6] and applying the corollaries of Theorem 3.6 appropriately, we prove Theorem 3.11. This theorem characterizes $\delta$-semistability for swamps in terms of GIT semistability on a particular parameter space that depends on two natural numbers, $N$ and $L$, that must be large enough. The novel part is that we prove that $N$ and $L$ do not depend on the base curve. From [14], the corresponding result for singular principal 
bundles follows, which, in turn, implies that the projective scheme we construct is a coarse moduli space for pairs $(X,(\mathscr{F}, \tau))$ given by a stable curve of genus $g$ and a $\delta$-semistable singular principal bundle.

\subsection{Outline of the Paper}

This paper is organized as follows. In Sect. 2, we introduce the notions of swamps and singular principal bundles, their relation, and the concept of $\delta$-semistability. In Sect. 3 , we prove the main technical result of the article, which is Theorem 3.6. This allows us to prove Theorem 3.11, which eventually implies the main property of the projective scheme we construct. In Sect. 4, we prove the existence of a coarse projective moduli space for the moduli functor defined by pairs $(X,(\mathscr{F}, \phi)), X$ being a stable curve of genus $g$ and $(\mathscr{F}, \phi)$ a $\delta$-semistable swamp of a given Hilbert polynomial. The forgetful map defines a morphism between this moduli space and $\overline{\mathrm{M}}_{g}$. Finally, in Sect. 5, we prove the existence of a coarse projective moduli space for the moduli functor defined by pairs $(X,(\mathscr{F}, \tau)), X$ being a stable curve of genus $g$, and $(\mathscr{F}, \tau)$ a $\delta$-semistable singular principal $G$-bundle of a given rank and degree 0 . As in the case of swamps, there is a morphism to $\overline{\mathrm{M}}_{g}$. This, together with the results given in $[14,20,24,25]$ and Sect. 3, implies that the fibers of the above morphism over smooth curves are precisely the classical moduli spaces of principal $G$-bundles constructed by A. Ramanathan.

\section{Preliminaries}

Let $\mathbb{C}$ be an algebraically closed field of characteristic 0 . We work in the category of schemes over $\mathbb{C}$.

Let $X$ be a Cohen-Macaulay projective and connected curve of genus $g$ together with a very ample invertible sheaf $\mathscr{O}_{X}(1)$.

Set $k \in \mathbb{Z}$. A coherent sheaf $\mathscr{F}$ on $X$ is $k$-regular if $H^{1}(X, \mathscr{F}(k-1))=0$. If $\mathscr{F}$ is $k$-regular and $k^{\prime}>k$, then $\mathscr{F}$ is also $k^{\prime}$-regular. From Serre's vanishing theorem, it follows that there is always an integer $k$, such that $\mathscr{F}$ is $k$-regular. The regularity of $\mathscr{F}$ is defined as $\operatorname{reg}(\mathscr{F}):=\inf \{k \in \mathbb{Z}: \mathscr{F}$ is $k$-regular $\}$.

Let $\mathscr{F}$ be a coherent sheaf on $X$. Its (polarized) degree and its (polarized) slope are defined as $\operatorname{deg}(\mathscr{F}):=\chi(\mathscr{F})-r \chi\left(\mathscr{O}_{X}\right), \mu(\mathscr{F}):=\chi(\mathscr{F}) / \alpha, \alpha$ being the multiplicity of $\mathscr{F}$ (the degree one coefficient of its Hilbert polynomial $P_{\mathscr{F}}$ ) and $r=\alpha / h$ its rank. If $X$ has irreducible components $X_{1}, \ldots, X_{l}$, and $\mu_{i}$ denotes the generic point of $X_{i}$, the multirank of $\mathscr{F}$ is the tuple $\left(\operatorname{dim}\left(\mathscr{F}_{\mu_{i}}\right)\right)_{i=1, \ldots, l}$. It has uniform rank $r$ if $\operatorname{dim}\left(\mathscr{F}_{\mu_{i}}\right)=r$ for each $i$.

A coherent sheaf on $X$ is of pure dimension one if $\operatorname{dim}(\operatorname{Supp}(\mathscr{G}))=1$ for every $\mathscr{G} \subseteq \mathscr{F}$. Recall that a coherent sheaf of pure dimension one, $\mathscr{F}$, is semistable if for any subsheaf $\mathscr{F}^{\prime} \subset \mathscr{F}, \mu\left(\mathscr{F}^{\prime}\right) \leq \mu(\mathscr{F})$. Recall also that for any coherent sheaf of pure dimension one, $\mathscr{F}$, there is a unique filtration (Harder-Narasimhan filtration) $0=\mathscr{F}_{0} \subset \mathscr{F}_{1} \subset \cdots \subset \mathscr{F}_{k}=\mathscr{F}$, such that the quotients $\mathscr{F}_{i} / \mathscr{F}_{i-1}$ are semistable sheaves with decreasing slopes. As usual, we will use the following notation: 


$$
\mu_{\max }(\mathscr{F}):=\max \left\{\mu\left(\mathscr{F}_{i} / \mathscr{F}_{i-1}\right) \mid i=1, \ldots, k\right\}=\mu\left(\mathscr{F}_{1}\right) .
$$

For any subsheaf $\mathscr{G} \subset \mathscr{F}$, we have $\mu(\mathscr{G}) \leq \mu_{\max }(\mathscr{F})$.

\subsection{Swamps}

Let $a, b \in \mathbb{N}$ be natural numbers, $P$ a polynomial with integral coefficients of degree one, and $\mathscr{D}$ an invertible sheaf over $X$.

Definition 2.1. A swamp over $X$ of type $(a, b, \mathscr{D})$ and Hilbert polynomial $P$ is a pair $(\mathscr{F}, \phi)$, where $\mathscr{F}$ is a coherent $\mathscr{O}_{X}$-module with Hilbert polynomial $P$ and $\phi:\left(\mathscr{F}^{\otimes a}\right)^{\oplus b} \rightarrow \mathscr{D}$ is a non-zero morphism of $\mathscr{O}_{X}$-modules.

Let $T$ be a scheme and $\pi_{X}: X \times T \rightarrow X$, and $\pi_{T}: X \times T \rightarrow T$ the first and the second projections, respectively. A family of swamps over $X$ of type $(a, b, \mathscr{D})$ parametrized by $T$ is a tuple $\left(\mathscr{F}_{T}, \phi_{T}, \mathscr{N}\right)$, where $\mathscr{F}_{T}$ is a coherent $\mathscr{O}_{X \times T}$-module flat over $T, \mathscr{N}$ is an invertible sheaf over $T$ and $\phi_{T}:\left(\mathscr{F}_{T}^{\otimes a}\right)^{\oplus b} \rightarrow \pi_{X}^{*} \mathscr{D} \otimes \pi_{T}^{*} \mathscr{N}$ is a morphism of $\mathscr{O}_{X \times T}$-modules. An isomorphism between families $\left(\mathscr{F}_{T}, \phi_{T}, \mathscr{N}\right)$ and $\left(\mathscr{F}_{T}^{\prime}, \phi_{T}^{\prime}, \mathscr{N}^{\prime}\right)$ is a pair of isomorphisms $f: \mathscr{F} \simeq \mathscr{F}^{\prime}, g: \mathscr{N} \simeq \mathscr{N}^{\prime}$, such that $\phi_{T}^{\prime} \circ\left(f^{\otimes a}\right)^{\oplus b}=\mathrm{id} \otimes \pi_{T}^{*}(g)$ (see [7]).

Let $\mathscr{F}$ be a coherent sheaf of pure dimension one over $X$. A weighted filtration of $\mathscr{F}$ is a filtration $\mathscr{F}_{\bullet} \equiv(0) \subset \mathscr{F}_{1} \subset \cdots \subset \mathscr{F}_{t} \subset \mathscr{F}_{t+1}=\mathscr{F}_{\text {, }}$ equipped with positive rational numbers $\underline{m}=\left(m_{1} \ldots, m_{t}\right) \in \mathbb{Q}_{>0}^{t}$.

Let $\phi:\left(\mathscr{F}^{\otimes a}\right)^{\oplus b} \rightarrow \mathscr{D}$ be a swamp on $X$ and let $\left(\mathscr{F}_{\bullet}, \underline{m}\right)$ be a weighted filtration. We denote by $\alpha_{i}$ the multiplicity of $\mathscr{F}_{i}$ and by $\alpha$ the multiplicity of $\mathscr{F}$. Let us define $\Gamma:=\sum_{1}^{t} m_{i} \Gamma^{\left(\alpha_{i}\right)}, \Gamma^{(l)}:=\left(l-\alpha, . \times{ }^{l}, l-\alpha, l,{ }^{\times} . .{ }^{-} l, l\right)$. Let us denote by $J$ the set of multi-indices $I=\left(i_{1}, \ldots, i_{a}\right)$, such that $i_{j} \in$ $\{1, \ldots, t+1\}$. Let us define

$$
\mu\left(\mathscr{F}_{\bullet}, \underline{m}, \phi\right):=\min _{I \in J}\left\{\Gamma_{\alpha_{i_{1}}}+\cdots+\Gamma_{\alpha_{i_{a}}}|\phi|_{\left(\mathscr{F}_{i_{1}} \otimes \cdots \otimes \mathscr{F}_{i_{a}}\right) \oplus b} \neq 0\right\}
$$

$\Gamma_{j}$ being the $j$ th component of $\Gamma$.

Definition 2.2. Let $\delta \in \mathbb{Q}_{>0}$ be a positive rational number. A swamp $(\mathscr{F}, \phi)$ of type $(a, b, \mathscr{D})$ is $\delta$-(semi)stable if for each weighted filtration $\left(\mathscr{F}_{\bullet}, \underline{m}\right)$ the inequality $\sum_{1}^{t} m_{i}\left(\alpha P_{\mathscr{F}_{i}}-\alpha_{i} P_{\mathscr{F}}\right)+\delta \mu\left(\mathscr{F}_{\bullet}, \underline{m}, \phi\right)(\leq) 0$ holds.

Remark 2.3. (1) There is a positive integer $A$, depending only on the input data $P$ and $a$, which allows considering just weighted filtrations satisfying $m_{i}<A$ to check the $\delta$-semistability condition. To see this, note that a swamp is $\delta$-(semi)stable if and only if the $\delta$-(semi)stability condition holds for every integral weighted filtration, i.e., filtrations with integral weights. Now, the claim follows from [7, Lemma 1.4] changing ranks by multiplicities. Observe that the upper bound $A$ does not depend either on $b$ or on $\mathscr{D}$.

(2) Note that if $(\mathscr{F}, \phi)$ is $\delta$-semistable, then $\mathscr{F}$ is of pure dimension one.

\subsection{Singular Principal Bundles}

Let $G$ be a semisimple linear algebraic group and $\rho: G \hookrightarrow \operatorname{SL}(V)$ a faithful representation of dimension $r$. Let $P$ be a polynomial with integral coefficients of degree one. 
Definition 2.4. A singular principal $G$-bundle over $X$ with Hilbert polynomial $P$ is a pair $(\mathscr{F}, \tau)$, where $\mathscr{F}$ is a coherent $\mathscr{O}_{X}$-module with Hilbert polynomial $P$ and $\tau: S^{\bullet}(V \otimes \mathscr{F})^{G} \rightarrow \mathscr{O}_{X}$ is a morphism of $\mathscr{O}_{X}$-algebras, which is not just the projection onto the zero-degree component.

A family of singular principal $G$-bundles parametrized by a scheme $T$, as well as an isomorphism between two families, is defined in the obvious way.

Consider a singular principal $G$-bundle on $X, \tau: S^{\bullet}(V \otimes \mathscr{F})^{G} \rightarrow \mathscr{O}_{X}$. We can find $s \in \mathbb{N}$, such that $S^{\bullet}(V \otimes \mathscr{F})^{G}$ is generated by the submodule $\bigoplus_{i=0}^{s} S^{i}(V \otimes \mathscr{F})^{G}$. Let $\underline{d} \in \mathbb{N}^{s}$ be so that $\sum i d_{i}=s$ !. Then, we have

$$
\bigotimes_{i=1}^{s}(V \otimes \mathscr{F})^{\otimes i d_{i}} \rightarrow \bigotimes_{i=1}^{s} S^{d_{i}}\left(S^{i}(V \otimes \mathscr{F})\right) \rightarrow \bigotimes_{i=1}^{s} S^{d_{i}}\left(S^{i}(V \otimes \mathscr{F})\right)^{G} \rightarrow \mathscr{O}_{X}
$$

Adding up these morphisms as $\underline{d} \in \mathbb{N}$ varies, we find a swamp (see [14] or [20])

$$
\operatorname{Swamp}(\tau):=\phi_{\tau}:\left((V \otimes \mathscr{F})^{\otimes s !}\right)^{\oplus N} \rightarrow \mathscr{O}_{X} .
$$

Let us define $a:=s !$ and $b:=N$. From [14, Theorem 5.5], we deduce that there is an $s \in \mathbb{N}$, which depends only on the numerical input data, large enough that the map

$$
\left\{\begin{array}{l}
\text { isomorphism classes of } \\
\text { singular principal } G \text {-bundles }
\end{array}\right\} \rightarrow\left\{\begin{array}{l}
\text { isomorphism classes of } \\
\text { swamps of type }\left(a, b, \mathscr{O}_{X}\right)
\end{array}\right\}
$$

is injective.

Definition 2.5. Let $\delta \in \mathbb{Q}_{>0}$ be a positive rational number. A singular principal $G$-bundle is said to be $\delta$-semi(stable) if its associated swamp is $\delta$ semi(stable).

\section{A Uniform Boundedness Result on Cohen-Macaulay Curves}

Although the aim is to construct moduli spaces over stable curves, the main results of this section hold for Cohen-Macaulay curves, so they are stated for this more general case.

\subsection{Locally Free Sheaves on the Projective Line}

Let $\mathscr{E}$ be a locally free sheaf of rank $r$ on the projective line $\mathbf{P}_{k}^{1}$. By $[8$, Théorème 2.1], there are integers $n_{1} \geq \cdots \geq n_{r}$, such that $\mathscr{E} \simeq \bigoplus_{i=1}^{r} \mathscr{O}_{\mathbf{P}_{k}^{1}}\left(n_{i}\right)$. The tuple $\left(n_{1}, \ldots, n_{r}\right)$ is defined as the type of $\mathscr{E}$ and is denoted by $\tau(\mathscr{E})$. We will denote by $\tau_{\min }(\mathscr{E})$ (respectively, $\tau_{\max }(\mathscr{E})$ ) the minimum (respectively, maximum) integer of the type $\tau(\mathscr{E})$ of $\mathscr{E}$, that is, $\tau_{\min }(\mathscr{E})=n_{r}$ (respectively $\left.\tau_{\max }(\mathscr{E})=n_{1}\right)$.

Let $r, m \in \mathbb{N}$ and $d \in \mathbb{Z}$ be integers. There are finitely many isomorphism classes of locally free sheaves on $\mathbf{P}^{1}$ of rank $r$, degree $d$, and $h^{0}\left(\mathbf{P}^{1}, \mathscr{E}\right)=m$. 
Such isomorphism classes are determined by the tuples of integers $n_{1} \geq \cdots \geq$ $n_{r}$, verifying the equations

$$
\begin{aligned}
\delta\left(n_{1}\right)+\cdots+\delta\left(n_{r}\right) & =m, \text { where } \delta\left(n_{i}\right)= \begin{cases}n_{i}+1 & \text { if } n_{i} \geq 0 \\
0 & \text { otherwise }\end{cases} \\
n_{1}+\cdots+n_{r} & =d .
\end{aligned}
$$

Let us denote by $N(r, d, m)$ the set of tuples of integers $n_{1} \geq \cdots \geq n_{r}$ satisfying $(5)$ and by $S_{-}(r, d, m)$ (resp. $\left.S_{+}(r, d, m)\right)$ the minimum (resp. maximum) among the integers $i \in \mathbb{Z}$ that appear as the smallest (resp. largest) integer in a tuple of $N(r, d, m)$. Therefore, any integer $n \in \mathbb{Z}$ that appears in a tuple $\left(n_{1}, \ldots, n_{r}\right) \in N(r, d, m)$ satisfies that $S_{+}(r, d, m) \geq n \geq S_{-}(r, d, m)$.

Lemma 3.1. Let $\mathscr{E}$ be a locally free sheaf of rank $r$, degree $d$ and $h^{0}\left(\mathbf{P}^{1}, \mathscr{E}\right)=$ $m$. Then, for every $n \geq-S_{-}(r, d, m), \mathscr{E}(n)$ is generated by its global sections and $h^{1}\left(\mathbf{P}^{1}, \mathscr{E}(n)\right)=0$.

Proof. Let $\tau(\mathscr{E})=\left(n_{1}, \ldots, n_{r}\right)$ be the type of $\mathscr{E}$, that is, $\mathscr{E}=\bigoplus_{i=1}^{r} \mathscr{O}_{X}\left(n_{i}\right)$. Then, $\mathscr{E}(n)$ is generated by global sections if and only if $n+n_{i} \geq 0$ for all $i$, that is, if and only if $n \geq-n_{i}$ for all $i$. However, $n_{i} \geq S_{-}(r, d, m)$ for every $i$ by definition, so taking $n \geq-S_{-}(r, d, m)$, we get the desired result. On the other hand, $h^{1}\left(\mathbf{P}^{1}, \mathscr{E}(n)\right)=\sum_{i=1}^{r} h^{0}\left(\mathbf{P}^{1}, \mathscr{O}_{\mathbf{P}^{1}}\left(-2-n_{i}-n\right)\right)$, and $h^{0}\left(\mathbf{P}^{1}, \mathscr{O}_{\mathbf{P}^{1}}\left(-2-n_{i}-n\right)\right)=0$ for every $n \geq-S_{-}(r, d, m)$, so we are done.

\subsection{Uniform Boundedness}

Let $X$ be a Cohen-Macaulay projective and connected curve together with a very ample invertible sheaf $\mathscr{O}_{X}(1)$ of degree $h$. By [16, Proposition 6], there exists a finite surjective morphism $f: X \rightarrow \mathbf{P}^{1}$, such that $f^{*} \mathscr{O}_{\mathbf{P}^{1}}(1) \simeq \mathscr{O}_{X}(1)$, and [12, Theorem 23.1] implies that $f$ is flat. Let $\mathscr{L}$ be a coherent sheaf over $X$ of degree $d$ and multiplicity $\alpha$, and define $\mathscr{E}:=f_{*} \mathscr{L}$. Since $f$ is finite and $f^{*} \mathscr{O}_{\mathbf{P}^{1}}(1) \simeq \mathscr{O}_{X}(1)$, we know that $P_{\mathscr{E}}(n)=P_{\mathscr{L}}(n)$. Assuming $n \gg 0$, we get

$$
P_{\mathscr{E}}(n)=\operatorname{rk}(\mathscr{E}) \cdot n+\operatorname{rk}(\mathscr{E})+\operatorname{deg}(\mathscr{E}), P_{\mathscr{L}}(n)=\alpha \cdot n+\frac{\alpha}{h}(1-g)+d ;
$$

hence, $\operatorname{rk}(\mathscr{E})=\alpha$ and $\operatorname{deg}(\mathscr{E})=(1-g) \frac{\alpha}{h}-\alpha+d$.

Assume now that $\mathscr{L}$ is an invertible sheaf. Then, $\mathscr{E}$ is locally free and there are integers $a_{1}(f) \geq \cdots \geq a_{h}(f)$, such that

$$
\mathscr{E}=f_{*} \mathscr{L}=\bigoplus_{i=1}^{h} \mathscr{O}_{\mathbf{P}_{k}^{1}}\left(a_{i}(f)\right), 1-g-h+d=\sum_{i=1}^{h} a_{i}(f) .
$$

Definition 3.2. Let $X, \mathscr{O}_{X}(1), f, \mathscr{L}$ be as above. We define the $f$-type of $\mathscr{L}$ as the tuple $\left(a_{1}(f), \ldots, a_{h}(f)\right)$, and it is denoted by $\tau_{f}(\mathscr{L})$.

Lemma 3.3. Fix $g, h, m \in \mathbb{N}$ and $d \in \mathbb{Z}$. There are integers $S_{-}, S_{+}$depending only on $g, h, m, d$, such that for any Cohen-Macaulay projective and connected curve of genus $g$ with a very ample invertible sheaf $\mathscr{O}_{X}(1)$ of degree $h$, any invertible sheaf $\mathscr{L}$ on $X$ of degree $d$ and $h^{0}(X, \mathscr{L})=m$, and any finite morphism $f: X \rightarrow \mathbf{P}^{1}$, such that $f^{*} \mathscr{O}(1) \simeq \mathscr{O}_{X}(1)$, the following holds:

$$
S_{+} \geq \tau_{f, \max }(\mathscr{E}), \tau_{f, \min }(\mathscr{E}) \geq S_{-} \text {, where } \mathscr{E}:=f_{*}(\mathscr{L}) \text {. }
$$


Furthermore, for every $n \geq-S_{-}, \mathscr{L}(n)$ is generated by its global sections and $h^{1}(X, \mathscr{L}(n))=0$.

Proof. By $(6), \operatorname{rk}(\mathscr{E})=h$ and $\operatorname{deg}(\mathscr{E})=1-g-h+d$. On the other hand, $h^{0}\left(\mathbf{P}^{1}, \mathscr{E}\right)=h^{0}(X, \mathscr{L})=m$. Then, $S_{-}:=S_{-}(h, 1-g-h+d, m)$ and $S_{+}:=$ $S_{+}(h, 1-g-h+d, m)$ satisfy the inequality given in (7). Besides, if $n \geq-S_{-}$, $\mathscr{E}(n)$ is generated by global sections and $h^{1}\left(\mathbf{P}^{1}, \mathscr{E}(n)\right)=0$ by Lemma 3.1. This implies that $h^{1}(X, \mathscr{L}(n))=0$, since $h^{1}(X, \mathscr{L}(n))=h^{1}\left(\mathbf{P}^{1}, \mathscr{E}(n)\right)$ and that $\mathscr{L}(n)$ is generated by its global sections, since the adjunction map, $f^{*} f_{*} \rightarrow$ id, is surjective for finite morphisms.

Lemma 3.4. Fix $g, h \in \mathbb{N}$ and $n \in \mathbb{Z}$. There exists a constant $C \in \mathbb{Z}$, depending only on $g, h, n$, such that for every Cohen-Macaulay projective and connected curve $X$ of genus $g$ with a very ample invertible sheaf $\mathscr{O}_{X}(1)$ of degree $h$, we have $\mu_{\max }(\mathscr{H}) \leq C$, where $\mathscr{H}=\oplus_{i=1}^{r} \mathscr{O}_{X}\left(n_{i}\right)$ with $r$ any natural number and $n_{i} \leq n$.

Proof. Let $X$ and $\mathscr{H}$ be as in the statement. Let $f: X \rightarrow \mathbf{P}^{1}$ be a finite and surjective morphism, such that $f^{*} \mathscr{O}_{\mathbf{P}^{1}}(1) \simeq \mathscr{O}_{X}(1)$. Then, $f_{*} \mathscr{H}=\oplus_{i=1}^{r} \mathscr{E}\left(n_{i}\right)$ for certain natural numbers $n_{1}, \ldots, n_{r}$, where $\mathscr{E}=f_{*} \mathscr{O}_{X}=\bigoplus_{i=1}^{h} \mathscr{O}_{\mathbf{P}_{k}^{1}}\left(a_{i}(f)\right)$. By (6), we know that $\operatorname{rk}(\mathscr{E})=h$ and $\operatorname{deg}(\mathscr{E})=1-g-h$. Let $\mathscr{F} \subset \mathscr{H}$ be a subsheaf of multiplicity $\alpha$. Since $f_{*} \mathscr{F}$ is locally free, there are integers $t_{1}, \ldots, t_{\alpha}$, such that $f_{*} \mathscr{F}=\oplus_{i=1}^{\alpha} \mathscr{O}_{X}\left(t_{i}\right)$. Let $n^{\prime}$ be the maximum among $n_{1}, \ldots, n_{r}$. Since $f_{*} \mathscr{F} \subset f_{*} \mathscr{H}$, we deduce that $t_{i} \leq \tau_{\max }(\mathscr{E})+n^{\prime} \leq \tau_{\max }(\mathscr{E})+n$, which implies that $\operatorname{deg}\left(f_{*} \mathscr{F}\right) \leq \alpha\left(\tau_{\max }(\mathscr{E})+n\right)$. On the other hand, $\operatorname{deg}(\mathscr{F})=$ $\alpha+\operatorname{deg}\left(f_{*} \mathscr{F}\right)-(1-g) \alpha / h, \operatorname{so} \operatorname{deg}(\mathscr{F}) \leq \alpha\left(1+\tau_{\max }(\mathscr{E})+n\right)-(1-g) \alpha / h$ and, therefore, $\mu(\mathscr{F}) \leq 1+\tau_{\max }(\mathscr{E})+n-(1-g) 1 / h$. Finally, by Lemma 3.3 , there is a constant $S_{+}$, depending only on $g$ and $h$, such that $\tau_{\max }(\mathscr{E}) \leq S_{+}$. Therefore, $\mu(\mathscr{F}) \leq 1+S_{+}+n-(1-g) 1 / h=: C$.

Lemma 3.5. Let $\underline{P}$ be a finite set of polynomials of degree one with integral coefficients. There is a natural number $B_{\tau}$ depending only on $\underline{P}$, such that for every Cohen-Macaulay projective and connected curve with a very ample invertible sheaf $\mathscr{O}_{X}(1)$ and every coherent sheaf, $\mathscr{F}$, of pure dimension one with Hilbert polynomial in $\underline{P}$, we have $\operatorname{dim}\left(\mathscr{F}_{x} / \mathfrak{m}_{x} \mathscr{F}_{x}\right) \leq B_{\tau}$.

Proof. Let us define $B_{\tau}$ as the maximum of the leading coefficients of the polynomials in $\underline{P}$. Consider a finite surjective morphism $f: X \rightarrow \mathbf{P}^{1}$, such that $f^{*} \mathscr{O}_{\mathbf{P}^{1}}(1) \simeq \mathscr{O}_{X}(1)$. Since $f$ must be flat, $f_{*} \mathscr{F}$ is locally free of rank bounded from above by $B_{\tau}$. Since $\operatorname{dim}\left(\mathscr{F}_{x} / \mathfrak{m}_{x} \mathscr{F}_{x}\right) \leq \operatorname{rk}\left(f_{*} \mathscr{F}\right)$, we are done.

Theorem 3.6. Fix $g, h, C \in \mathbb{N}$ with $g \geq 2$, and a finite set $\underline{P}$ of polynomials of degree one with integral coefficients. There is a natural number $N_{0}$, depending only on $\underline{P}, C, g$, and $h$, such that for every Cohen-Macaulay projective and connected curve of genus $g$ with a very ample invertible sheaf $\mathscr{O}_{X}(1)$ of degree $h$ and every coherent sheaf of pure dimension one, $\mathscr{F}$, over $X$ with Hilbert polynomial in $\underline{P}$ satisfying $\mu_{\max }(\mathscr{F}) \leq C$, the following holds: for all $k \geq N_{0}$, $h^{1}(X, \mathscr{F}(k))=0$, and $\mathscr{F}(k)$ is generated by its global sections. 
Proof. Let $X, \mathscr{O}_{X}(1)$ and $\mathscr{F}$ be as in the statement. By Serre duality theorem, we have that $h^{1}(X, \mathscr{F}(k))=\operatorname{dim}\left(\operatorname{Hom}_{\mathscr{O}_{X}}\left(\mathscr{F}(k), \omega_{X}\right)\right), \forall k \in \mathbb{Z}$. Suppose that $h^{1}(X, \mathscr{F}(k)) \neq 0$ and $k \geq 0$. Then, there is a non-zero morphism $f^{\prime}: \mathscr{F} \rightarrow$ $\omega_{X}(-k)$. Let us define $\mathscr{K}$ and $\mathscr{N}$ to be $\operatorname{Ker}\left(f^{\prime}\right)$ and $\operatorname{Im}\left(f^{\prime}\right)$, respectively. From the exact sequence $0 \rightarrow \mathscr{K} \rightarrow \mathscr{F} \rightarrow \mathscr{N} \rightarrow 0$, we get

$$
\begin{aligned}
\operatorname{deg}(\mathscr{N}) & =\operatorname{deg}(\mathscr{F})-\operatorname{deg}(\mathscr{K})=\operatorname{deg}(\mathscr{F})-\alpha_{\mathscr{K}} \mu(\mathscr{K}) \geq \operatorname{deg}(\mathscr{F})-\alpha_{\mathscr{K}} C \\
& =P_{\mathscr{F}}(0)-\frac{P_{\mathscr{F}}^{\prime}(n)}{h}(1-g)-\alpha_{\mathscr{K}} C \geq B_{0},
\end{aligned}
$$

with $B_{0}:=\min \left\{P_{\mathscr{F}}(0)-\frac{P_{\mathscr{F}}^{\prime}(n)}{h}(1-g)-\left.i \cdot C\right|_{i} \in\left[1, \alpha_{\max }\right], P_{\mathscr{F}}(n) \in \underline{P}\right\}$. Here, $\alpha_{\max }$ is the maximum among the degree one coefficients of the polynomials in $\underline{P}$. Note that $B_{0}$ is a constant which depends only on $\underline{P}, h$ and the genus $g$. The injective morphism $\mathscr{N} \hookrightarrow \omega_{X}(-k)$ induces an injective morphism $\mathscr{N}(k) \hookrightarrow \omega_{X}$. Then, we have $\operatorname{deg}(\mathscr{N}(k))=\alpha_{\mathscr{N}} \cdot k+\operatorname{deg}(\mathscr{N}) \geq k+B_{0}$, and therefore

$$
\begin{aligned}
2 g-2 & =\operatorname{deg}\left(\omega_{X}\right)=\operatorname{deg}(\mathscr{N}(k))+\operatorname{deg}\left(\omega_{X} / \mathscr{N}(k)\right) \\
& \geq k+B_{0}+\operatorname{deg}\left(\omega_{X} / \mathscr{N}(k)\right) .
\end{aligned}
$$

Let us find a bound of $\operatorname{deg}\left(\omega_{X} / \mathscr{N}(k)\right)$. Denote by $\mathscr{J}$ the quotient $\omega_{X} / \mathscr{N}(k)$. Fix a finite surjective morphism $f: X \rightarrow \mathbf{P}^{1}$, such that $f^{*} \mathscr{O}_{\mathbf{P}^{1}}(1) \simeq \mathscr{O}_{X}(1)$. Since $f$ is finite, we have a surjection $f_{*}\left(\omega_{X}\right) \rightarrow f_{*} \mathscr{J} \rightarrow 0$. Denote by $\mathscr{T}$ the torsion subsheaf of $f_{*} \mathscr{J}$ and by $\mathscr{U}=f_{*} \mathscr{J} / \mathscr{T}$ the locally free subsheaf. If $\mathscr{U}=0, \operatorname{deg}\left(f_{*} \mathscr{I}\right) \geq 0$. On the other hand, if $\mathscr{U} \neq 0$, we have $f_{*} \mathscr{J}=\mathscr{U} \oplus \mathscr{T}$, so $\operatorname{deg}\left(f_{*} \mathscr{J}\right) \geq \operatorname{deg}(\mathscr{U})$, and it is enough to give a bound of $\operatorname{deg}(\mathscr{U})$. Observe that we have a surjective morphism $f_{*}\left(\omega_{X}\right) \rightarrow \mathscr{U} \rightarrow 0$, and that

$$
f_{*}\left(\omega_{X}\right)=\bigoplus_{i=1}^{h} \mathscr{O}\left(a_{i}\right), \quad \mathscr{U}=\bigoplus_{i=1}^{T} \mathscr{O}\left(b_{j}\right) .
$$

From the above surjection, it follows that, given an index $j=1, \ldots T$, there exists an index $i=1, \ldots h$ such that $b_{j} \geq a_{i}$. Therefore

$$
\operatorname{deg}(\mathscr{U})=\sum_{i=1}^{T} b_{i} \geq \sum_{l=1}^{T} a_{i_{k}} \geq T \cdot A,
$$

where $A:=\min \left\{a_{i}\right\}$. Now, by Proposition 3.3, there exists an integer $S=$ $S(g, h, m)$ depending only on $g, h, m:=h^{0}\left(X, \omega_{X}\right)=g$ such that $A \geq S$. Therefore, $\operatorname{deg}(\mathscr{U}) \geq T \cdot S \geq T_{0} \cdot S$, where $T_{0}:=\min \{0, h \cdot S\}$. Set $C_{0}=$ $\min \left\{T_{o} \cdot S, 0\right\}$. Then, from (8), we get

$$
2 g-2 \geq k+B_{0}+C_{0} .
$$

Let $N_{0}^{\prime}$ be the smallest integer, such that $N_{0}^{\prime}+B_{0}+C_{0}>2 g-2$. Then, $h^{1}(X, \mathscr{F}(k))=0$ for all $k \geq N_{0}^{\prime}$ and $N_{0}^{\prime}$ only depends on $P, C, g, h$.

For the last part, let $x \in X$ be a closed point and $\mathscr{I}_{x}$ its ideal sheaf. Let us define $\mathscr{G}:=\operatorname{Im}\left(\mathscr{F} \otimes_{\mathscr{O}_{X}} \mathscr{I}_{x} \rightarrow \mathscr{F}\right)$ and $d(x):=\operatorname{dim} \mathscr{F}_{x} / \mathfrak{m}_{x} \mathscr{F}_{x}$. We have $\mu_{\max }(\mathscr{G}) \leq C$ and $P_{\mathscr{G}}(n)=P_{\mathscr{F}}(n)-d(x)$. By Lemma 3.5, the function $d(x)$ is bounded from above by a constant $B_{\tau}$ that depends only on $\underline{P}$. We can argue now as above and we arrive at the equation $2 g-2 \geq k+B_{0}-B_{\tau}+C_{0}$. Let 
$N_{0}\left(\geq N_{0}^{\prime}\right)$ be the smallest integer such that $N_{0}+B_{0}-B_{\tau}+C_{0}>2 g-2$. Then, $h^{1}(X, \mathscr{F}(-x)(k))=h^{1}(X, \mathscr{F}(k))=0$ for all $k \geq N_{0}$, so $\mathscr{F}(k)$ is generated by its global sections and $h^{1}(X, \mathscr{F}(k))=0$ for every $k \geq N_{0}$ and $N_{0}$ only depends on $\underline{P}, C, g, h$.

Corollary 3.7. Fix $g, h, C, C^{\prime}, a \in \mathbb{N}$ with $g \geq 2$, and a finite set $\underline{P}$ of polynomials of degree one with integral coefficients. There are natural numbers $N_{1}, N_{1}^{\prime}$ depending on $g, h, C, C^{\prime}, \underline{P}$ and $g, h, C, a, \underline{P}$, respectively, such that for every Cohen-Macaulay projective and connected curve of genus $g$ with a very ample invertible sheaf $\mathscr{O}_{X}(1)$ of degree $h$, and every family $E$ of equivalence classes of coherent sheaves of pure dimension one, $\mathscr{F}$, with Hilbert polynomial in $\underline{P}$ that satisfy $\mu_{\max }(\mathscr{F}) \leq C$, the following holds:

(1) For every subsheaf $\mathscr{F}^{\prime} \subset \mathscr{F}$, with $\mathscr{F} \in E$, such that $\left|\operatorname{deg}\left(\mathscr{F}^{\prime}\right)\right| \leq C^{\prime}$, we have $h^{1}\left(X, \mathscr{F}^{\prime}(k)\right)=0$ and $\mathscr{F}^{\prime}(k)$ is generated by its global sections for all $k \geq N_{1}$.

(2) For every sheaf of the form $\mathscr{H}=\mathscr{F}_{1} \otimes \cdots \otimes \mathscr{F}_{a}$ with $\mathscr{F}_{i} \in E$, we have $h^{1}(X, \mathscr{H}(k))=0$ and $\mathscr{H}(k)$ is generated by its global sections for all $k \geq N_{1}^{\prime}$.

Proof. 1. Let $\mathscr{F} \in E$ and $\mathscr{F}^{\prime} \subset \mathscr{F}$ a coherent sheaf in $E^{\prime}$. We have $\mu_{\max }\left(\mathscr{F}^{\prime}\right) \leq C$. Since $\left|\operatorname{deg}\left(\mathscr{F}^{\prime}\right)\right| \leq C^{\prime}$, there are only finitely many possible polynomials in the set of Hilbert polynomials of the members of $E^{\prime}$. Then, applying Theorem 3.6, we conclude.

2. By Theorem 3.6, it is enough to show that if $\mathscr{F}, \mathscr{G}$ are coherent sheaves of $\mathscr{O}_{X}$-modules that are $k_{1}$ - and $k_{2}$-regular, respectively, then $\mathscr{F} \otimes \mathscr{G}$ is $\left(k_{1}+k_{2}\right)$-regular. Let $j: X \hookrightarrow \mathbb{P}^{n}$ be a closed immersion, such that $j^{*} \mathscr{O}_{\mathbb{P}^{n}}(1)=\mathscr{O}_{X}(1)$. Since $j: X \hookrightarrow \mathbb{P}^{n}$ is a finite morphism, we know that $H^{i}(X, \mathscr{F}(n))=H^{i}\left(\mathbb{P}^{n}, j_{*}(\mathscr{F})(n)\right)$ and $H^{i}(X, \mathscr{G}(n))=$ $H^{i}\left(\mathbb{P}^{n}, j_{*}(\mathscr{G})(n)\right)$. This implies that $j_{*} \mathscr{F}$ and $j_{*} \mathscr{G}$ are $k_{1^{-}}$and $k_{2}$-regular, respectively, and obviously, $\left(j_{*} \mathscr{F}\right)_{y}=\left(j_{*} \mathscr{G}\right)_{y}=0$ for every $y \in \mathbb{P}^{n} \backslash X$. Therefore, by [28, Proposition 1.5], $j_{*} \mathscr{F} \otimes j_{*} \mathscr{G}$ is $\left(k_{1}+k_{2}\right)$-regular. That is, $H^{1}\left(\mathbb{P}^{n},\left(j_{*} \mathscr{F} \otimes j_{*} \mathscr{G}\right)\left(k_{1}+k_{2}-1\right)\right)=0$ for every $i \geq 0$. Since $j$ is a closed immersion, we deduce that $j_{*} \mathscr{F} \otimes j_{*} \mathscr{G}=j_{*}(\mathscr{F} \otimes \mathscr{G})$ and, therefore, $h^{1}\left(X,(\mathscr{F} \otimes \mathscr{G})\left(k_{1}+k_{2}-1\right)\right)=h^{1}\left(\mathbb{P}^{n},\left(j_{*} \mathscr{F} \otimes j_{*} \mathscr{G}\right)\left(k_{1}+k_{2}-1\right)\right)=0$.

Corollary 3.8. Let $F$ be a finite-dimensional vector space, $k \in \mathbb{Z}, l_{0} \in \mathbb{N}$, $P(n) \in \mathbb{Z}[n]$, and $X$ a Cohen-Macaulay projective and connected curve of genus $g \geq 2$ with a very ample invertible sheaf $\mathscr{O}_{X}(1)$ with $h^{1}\left(X, \mathscr{O}_{X}\left(l_{0}\right)\right)=0$. Then, there exists a natural number $L \in \mathbb{Z}$ depending only on $k, l_{0}, g, P(n)$, such that for every quotient sheaf $q: F \otimes \mathscr{O}_{X}(k) \rightarrow \mathscr{F} \rightarrow 0$ with Hilbert polynomial $P(n)$ and for every vector subspace $F^{\prime} \subset F$, the following holds: $h^{1}\left(X, \mathscr{F}_{F^{\prime}}(l)\right)=0$ and $H^{0}(q(l))\left(F^{\prime} \otimes W\right)=H^{0}\left(X, \mathscr{F}_{F^{\prime}}(l)\right)$ for every $l>L$, where $W=H^{0}\left(X, \mathscr{O}_{X}(l+k)\right)$ and $\mathscr{F}_{F^{\prime}}$ is the subsheaf $q\left(F^{\prime} \otimes \mathscr{O}_{X}(k)\right) \subset \mathscr{F}$.

Proof. Let $X$ be a Cohen-Macaulay projective and connected curve of genus $g \geq 2$ together with a very ample invertible sheaf $\mathscr{O}_{X}(1)$ with the conditions of the statement, let $q: F \otimes \mathscr{O}_{X}(k) \rightarrow \mathscr{F} \rightarrow 0$ be a quotient sheaf with Hilbert polynomial $P(n)$, and let $F^{\prime} \subset F$ be a vector subspace. Let us consider the 
exact sequence $0 \rightarrow \mathscr{K}_{F^{\prime}}(l) \rightarrow F^{\prime} \otimes \mathscr{O}_{X}(l+k) \rightarrow \mathscr{F}_{F^{\prime}}(l) \rightarrow 0$. Taking global sections, we get a surjection $F^{\prime} \otimes H^{1}\left(X, \mathscr{O}_{X}(l+k)\right) \rightarrow H^{1}\left(X, \mathscr{F}_{F^{\prime}}(l)\right) \rightarrow$ 0 . Clearly, $h^{1}\left(X, \mathscr{O}_{X}(p)\right)=0$ for every $p \geq l_{0}$, since $h^{1}\left(X, \mathscr{O}_{X}\left(l_{0}\right)\right)=0$. Therefore, $l>-k+l_{0}$ implies $h^{1}\left(X, \mathscr{F}_{F^{\prime}}(l)\right)=0$. On the other hand, it follows from Lemma 3.4 that every sheaf $\mathscr{K}$ of the form $\operatorname{Ker}(q)$, for some $q: F \otimes \mathscr{O}_{X}(k) \rightarrow \mathscr{F}$ as in the statement, satisfies that $\mu_{\max }(\mathscr{K})$ is bounded from above by a constant depending only on the numerical input data. Also, there is a finite set $\underline{P}$ of polynomials with integral coefficients, depending only on the numerical input data as well, to which $P_{\mathscr{H}}(n)$ belongs. Now, by Theorem 3.6, we deduce that there is a constant $l_{1} \in \mathbb{N}$, depending only on the numerical input data, such that for every $l \geq l_{1}, h^{1}\left(X, \mathscr{K}_{F^{\prime}}(l)\right)=0$ and $\mathscr{K}_{F^{\prime}}(l)$ is generated by its global sections. Thus, for every $l \geq L:=$ $\max \left\{-k+l_{0}, l_{1}\right\}$, we have $H^{0}(q(l))\left(F^{\prime} \otimes W\right)=H^{0}\left(X, \mathscr{F}_{F^{\prime}}(l)\right)$.

\section{3. $\delta$-Semistable Swamps}

A direct consequence of Theorem 3.6 is that there is a bound of the regularity of coherent $\mathscr{O}_{X}$-modules appearing in $\delta$-semistable swamps (and, therefore, in $\delta$-semistable singular principal bundles) that works for every curve in $\overline{\mathrm{M}_{g}}$. This result is necessary to find a universal parameter space over $\overline{\mathrm{M}}_{g}$ for such objects.

Theorem 3.9. Let $a, g, h \in \mathbb{N}$ with $g \geq 2$ and let $P(n) \in \mathbb{Z}[n]$ be a polynomial of degree one and $\delta \in \mathbb{Q}_{>0}$. There exists a natural number $N_{2} \in \mathbb{N}$ depending only on $P(n), a, \delta, g, h$, such that for every Cohen-Macaulay projective and connected curve of genus $g, X$, with very ample invertible sheaf $\mathscr{O}_{X}(1)$ of degree $h$ and for every coherent sheaf of pure dimension one $\mathscr{F}$ with Hilbert polynomial $P(n)$ appearing in a $\delta$-(semi)stable swamp of type $(a,-,-)$, the following holds: $\mathscr{F}(k)$ is generated by its global sections and $h^{1}(X, \mathscr{F}(k))=0$ for every $k \geq N_{2}$.

Remark 3.10. The notation $(a,-,-)$ means that we allow swamps of type $(a, b, \mathscr{D})$, whatever $b \in \mathbb{N}$ and $\mathscr{D} \in \operatorname{Pic}(X)$ are.

Proof. Let $(\mathscr{F}, \phi)$ be a $\delta$-semistable swamp of type $(a, b, \mathscr{D})$ with Hilbert polynomial $P(n)$. Let $\mathscr{F}_{1} \subset \mathscr{F}$ be a subsheaf and consider the one-step flag $0 \subset \mathscr{F}_{1} \subseteq \mathscr{F}_{2}=\mathscr{F}$. The computation of the semistability condition given in Definition 2.2 leads to the inequality

$$
\mu\left(\mathscr{F}_{1}\right) \leq C:=\mu(\mathscr{F})+\frac{a(\alpha-1)}{\alpha} \delta,
$$

$C$ being a constant depending only on $P, h, a, \delta, g$. Now, by Theorem 3.6, there exists a natural number $N_{2} \in \mathbb{N}$ depending only on $P(n), C, g, h$ (thus, on $P(n), a, \delta, g, h)$, such that $h^{1}(X, \mathscr{F}(k))=0$ and $\mathscr{F}(k)$ is generated by its global sections for every $k \geq N_{2}$.

Let $a, b, g, h, l_{0} \in \mathbb{N}$ be natural numbers with $g \geq 2$, and let $P(n) \in \mathbb{Z}[n]$ be a polynomial of degree one and $\delta \in \mathbb{Q}_{>0}$. Let $X$ be a Cohen-Macaulay projective and connected curve of genus $g$ with a very ample invertible sheaf of degree $h$, such that $h^{1}\left(X, \mathscr{O}_{X}\left(l_{0}\right)\right)=0$ for a fixed natural number $l_{o} \in \mathbb{N}$. Let $D \in \mathbb{N}$ be a natural number, $e \in \mathbb{Z}$ an integer, and let $\mathscr{D}$ be an invertible 
sheaf on $X$ of degree $e$ with $h^{0}(X, \mathscr{D})=D$. Given $k \in \mathbb{N}$, let $\mathrm{Q}(X, k, P)$ denote

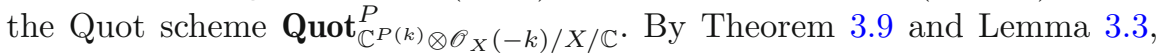
there is a natural number $N^{\prime} \in \mathbb{Z}$, depending only on the numerical input data, such that for every $k \geq N^{\prime}$ and for each $\delta$-(semi)stable swamp, $(\mathscr{F}, \phi)$, of type $(a, b, \mathscr{D})$ and Hilbert polynomial $P$, we have that $\mathscr{F}(k)$ and $\mathscr{D}(k)$ are generated by its global sections, and $h^{1}(X, \mathscr{F}(k))=h^{1}(X, \mathscr{D}(k))=0$. Fix such a natural number $k>N^{\prime}$ and denote by $F$ the vector space $\mathbb{C}^{P(k)}$. Let $L$ be as in Corollary 3.8. For $l \geq L$, there is a projective embedding (Grothendieck embedding composed with the Plücker embedding)

$$
\mathrm{Q}(X, k, P) \hookrightarrow \mathbf{P}\left(\bigwedge^{P(l)}\left(F \otimes H^{0}\left(X, \mathscr{O}_{X}(l-k)\right)\right)\right) .
$$

Consider now the functor ${ }^{\text {rig }} \mathbf{S w}_{P, \mathscr{D}, a, b}^{k}$ which assigns the set of isomorphism classes of tuples $\left(\mathscr{F}_{T}, \phi_{T}, \mathscr{N}, g_{T}\right)$ to a scheme $T$, where $\left(\mathscr{F}_{T}, \phi_{T}, \mathscr{N}\right)$ is a family of swamps with Hilbert polynomial $P$ and $g_{T}$ is a morphism $g_{T}: F \otimes \mathscr{O}_{T} \rightarrow$ $\pi_{T *} \mathscr{F}_{T}(k)$, such that the induced morphism $F \otimes \mathscr{O}_{X \times T} \rightarrow \mathscr{F}_{T}(k)$ is surjective. The functor ${ }^{r i g} \mathbf{S w}_{P, \mathscr{D}, a, b}^{k}$ is represented by a closed subscheme $W_{P, \mathscr{D}, a, b}^{k, l}(X)$ of $\mathrm{Q}(X, k, P) \times \mathbf{P}\left(Z_{1}\right)$, with $Z_{1}=\left(\left(F^{\otimes a}\right)^{\oplus b}\right)^{\vee} \otimes H^{0}(X, \mathscr{D}(a k))$ (see [7, Section $3]$ for smooth projective varieties and [13, Theorem 2.1.36] for nodal curves). Let $Z_{P, \mathscr{D}, a, b}^{k, l}(X) \subset W_{P, \mathscr{D}, a, b}^{k, l}(X)$ be the closure of the locus representing $\delta$ semistable swamps. Consider the projections $p_{Q}: Z_{P, \mathscr{D}, a, b}^{k, l}(X) \rightarrow \mathrm{Q}(X, k, P)$ and $p_{\mathbf{P}\left(Z_{1}\right)}: Z_{P, \mathscr{D}, a, b}^{k, l}(X) \rightarrow \mathbf{P}\left(Z_{1}\right)$, and define a polarization on $Z_{P, \mathscr{D}, a, b}^{k, l}(X)$ by $\mathscr{O}_{Z_{k, \mathscr{O}}(X)}\left(n_{1}, n_{2}\right):=p_{Q}^{*} \mathcal{O}_{\mathrm{Q}(X, k, P)}\left(n_{1}\right) \otimes p_{\mathbf{P}\left(Z_{1}\right)}^{*} \mathscr{O}_{\mathbf{P}\left(Z_{1}\right)}\left(n_{2}\right), n_{1}$ and $n_{2}$ being positive integers, such that

$$
\frac{n_{1}}{n_{2}}=\frac{P(l)-P(k)}{P(k)-a \delta} \delta .
$$

The natural action of $\mathrm{SL}(F)$ on $\mathrm{Q}(X, k, P) \times \mathbf{P}\left(Z_{1}\right)$ preserves $Z_{P, \mathscr{D}, a, b}^{k, l}(X)$ and the linearizations on $\mathscr{O}_{\mathbf{Q}(X, k, P)}(1)$ and $\mathscr{O}_{\mathbf{P}\left(Z_{1}\right)}(1)$ induce a linearization on the invertible sheaf $\mathscr{O}_{Z_{P, \mathscr{Q}, a, b}^{k, l}(X)}\left(n_{1}, n_{2}\right)$. Then, the GIT quotient given by

$$
\mathcal{T}_{P, \mathscr{D}, a, b}^{\delta-(\mathrm{s}) \mathrm{s}}(X):=Z_{P, \mathscr{D}, a, b}^{k, l}(X) / / \mathrm{SL}(F)
$$

exists and is projective, and if $k, l$ are large enough, it is a coarse moduli space of $\delta$-semistable swamps of the corresponding type (see [7, Theorem 1.8], [11, Theorem 3.5] and [13, Theorem 2.1.44]).

Now, we can show that we may choose $k$ and $l$, so that they work for every Cohen-Macaulay projective and connected curve of genus $g$. To do so, we need only to prove the following result.

Theorem 3.11. Let $g, h, D, a, b \in \mathbb{N}$ be natural numbers with $g \geq 2$ and $P(n) \in$ $\mathbb{Z}[n]$ a polynomial of degree one. There are natural numbers $N\left(\geq N^{\prime}\right), L \in \mathbb{N}$ depending only on the numerical input data, such that for every $k \geq N$ and every $l \geq L$, the following holds: for every Cohen-Macaulay projective and connected curve of genus $g$ with a very ample invertible sheaf $\mathscr{O}_{X}(1)$ of degree $h$, a point $(q, \Phi) \in Z_{P, \mathscr{D}, a, b}^{k, l}(X)$ is GIT-(semi)stable with respect 
to $\mathscr{O}_{Z_{P, \mathscr{D}, a, b}^{k, l}(X)}\left(n_{1}, n_{2}\right)$ if and only if the corresponding swamp $(\mathscr{F}, \phi)$ is $\delta$ (semi)stable and the linear map $f_{q}: F \rightarrow H^{0}(X, \mathscr{F}(k))$ is an isomorphism, where $F:=\mathbb{C}^{P(k)}$.

Remark 3.12. This result can be found in [7, Theorem 3.6] without the uniformity property for $N$ and $L$, in case $X$ is a smooth projective variety. The special case $\operatorname{dim}(X)=1$ can be generalized for reduced projective and connected curves [13, Theorem 2.1.41], and the proof is valid for a fixed CohenMacaulay projective and connected curve. It only remains to show that we can choose $N$ and $L$, so that they work for any curve. To avoid repetition, we will only mention at which steps (see [13, Section 2.1.5]) Theorem 3.6 and its corollaries must be applied to get the desired result.

Proof. First, a short calculation shows that the constants appearing in [13, Lemma 2.1.23] do not depend on the base curve. Apply Theorem 3.6 and Corollary 3.7 1) in [13, Lemma 2.1.24, Lemma 2.1.25]. The above, together with Corollary 3.72 ), allows us to show that the constant appearing in [13, Theorem 2.1.26] and [13, Corollary 2.1.27] does not depend on the base curve either. Corollary 3.8 implies that the constant appearing in the numerical criterion [13, Proposition 2.1.39] depends only on the numerical input data. Finally, applying the above results, it follows that the constant appearing in [13, Theorem 2.1.41] is, again, independent on the base curve.

\section{The Universal Moduli Space of Swamps}

Let $a, b, g \in \mathbb{N}$ be natural numbers with $g \geq 2, \delta \in \mathbb{Q}_{>0}$ a positive rational number, and $P$ a polynomial with integral coefficients of degree one. Consider the moduli functor given by

$$
\mathbf{S w}_{P, g, a, b}^{\delta \text { (s)s }}(T)=\left\{\begin{array}{l}
\text { isomorphism classes of pairs }\left(X_{T},\left(\mathscr{F}_{T}, \phi_{T}, \mathscr{N}\right)\right), \\
\text { where } X_{T} \text { is a stable curve of genus } g \text { flat over } \\
T \text { and }\left(\mathscr{F}_{T}, \phi_{T}, \mathscr{N}\right) \text { is a family of } \delta \text {-(semi)stable } \\
\text { swamps over } T \text { with Hilbert polynomial } P \text { and } \\
\text { type }\left(a, b, \mathscr{O}_{X_{T}}\right)
\end{array}\right\} .
$$

Two pairs $\left(X_{T},\left(\mathscr{F}_{T}, \phi_{T}, \mathscr{N}\right)\right)$ and $\left(X_{T}^{\prime},\left(\mathscr{F}_{T}^{\prime}, \phi_{T}^{\prime}, \mathscr{N}^{\prime}\right)\right)$ are isomorphic if there is an isomorphism of $T$-schemes, $u: X_{T} \simeq X_{T}^{\prime}$, such that $u^{*}\left(\mathscr{F}_{T}^{\prime}, \phi_{T}^{\prime}, \mathscr{N}^{\prime}\right)$ and $\left(\mathscr{F}_{T}, \phi_{T}, \mathscr{N}\right)$ are isomorphic (see Sect. 2.1).

Our goal is to prove the following theorem.

Theorem 4.1. There exists a projective scheme, $\mathcal{T}_{P, g, a, b}^{\delta-(\mathrm{s}) \mathrm{s}}$, and a morphism $\Theta_{s w}: \mathcal{T}_{P, g, a, b}^{\delta-(\mathrm{s}) \mathrm{s}} \rightarrow \overline{\mathrm{M}}_{g}$, such that $\Theta_{s w}^{-1}([X])=\mathcal{T}_{P, \mathscr{O}_{X}, a, b}^{\delta-(\mathrm{s}) \mathrm{s}}(X) / \operatorname{Aut}(X)$ for any stable curve $[X] \in \overline{\mathrm{M}}_{g}$.

\subsection{Gieseker Construction of $\overline{\mathbf{M}}_{g}$}

Let us summarize the construction of $\overline{\mathrm{M}}_{g}[6]$. Let $g \geq 2$ be a natural number and set $d:=10(2 g-2)$ and $M:=d-g$. These data determine a polynomial 
$h(s)=d s-g+1$. Consider the Hilbert scheme $\mathrm{H}_{d, g, M}$ representing projective curves of genus $g$ and degree $d$ in $\mathbf{P}^{M}$. There exists a projective embedding

$$
i_{s}: \mathrm{H}_{d, g, M} \hookrightarrow \operatorname{Grass}\left(h(s), H^{0}\left(\mathbf{P}^{M}, \mathcal{O}_{\mathbf{P}^{M}}(s)\right)^{\vee}\right) \hookrightarrow \mathbf{P}\left(H_{1}\right),
$$

with $H_{1}=\bigwedge^{h(s)} H^{0}\left(\mathbf{P}^{M}, \mathcal{O}_{\mathbf{P}^{M}}(s)\right)$, for each $s \in \mathbb{N}$ greater than a particular natural number $s_{0} \in \mathbb{N}$. Every pair given by a stable curve $X$ of genus $g$ and an isomorphism $\mathbb{C}^{M+1} \simeq H^{0}\left(X, \omega_{X}^{\otimes 10}\right)$ determines a point $[X] \in \mathrm{H}_{d, g, M}$. Let us denote by $\mathrm{H}_{g} \subset \mathrm{H}_{d, g, M}$ the quasi-projective variety of non-degenerate, 10-canonical stable curves of genus $g$. The action of $\mathrm{SL}_{M+1}$ on $\mathbf{P}^{M}$ induces an action on $\mathrm{H}_{g}$. Then, there exists a natural number $s_{1}$, such that for every $s \geq s_{1}$, the following holds: (1) $i_{s}\left(\mathrm{H}_{g}\right)$ belongs to the stable locus, and $(2)$ $i_{s}\left(\mathrm{H}_{g}\right)$ is closed in the semistable locus. It follows that $\overline{\mathrm{M}}_{g}=\mathrm{H}_{g} / \mathrm{SL}_{M+1}$ exists and is projective. The scheme $\mathrm{H}_{g}$ is endowed with a universal family, $\mu: U_{g} \rightarrow \mathrm{H}_{g}$, of genus $g$ stable curves and a diagram

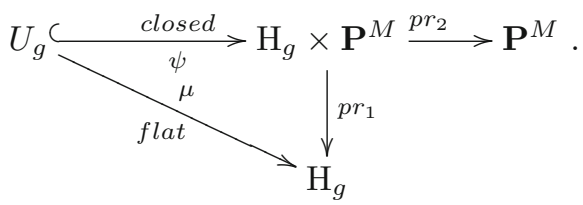

For any closed point $h \in \mathrm{H}_{g}, \psi$ induces a closed immersion $\psi_{h}: X_{h} \hookrightarrow \mathbf{P}^{M}$, $X_{h}$ being the fiber of $\mu$ over $h \in \mathrm{H}_{g}$, which satisfies that $\psi_{h}^{*} \mathscr{O}_{\mathbf{P}}{ }^{M}(1) \simeq \omega_{X_{h}}^{\otimes 10}$. Denote by $\nu$ the composition $p r_{2} \circ \psi: U_{g} \rightarrow \mathbf{P}^{M}$ and by $\mathscr{O}_{U_{g}}(1)$ the relatively very ample invertible sheaf $\nu^{*} \mathscr{O}_{\mathbf{P}^{M}}(1)$. Then, $\left.\mathscr{O}_{U_{g}}(1)\right|_{X_{h}} \simeq \omega_{X_{h}}^{\otimes 10}$ for every $h \in \mathrm{H}_{g}$. Since $h^{1}\left(X, \omega_{X}^{\otimes 10}\right)=0$ and $h^{0}\left(X, \omega_{X}^{\otimes 10}\right)=10(2 g-2)-g+1$ for every stable curve, we have that $R^{1} \mu_{*} \mathscr{O}_{U_{g}}(1)=0, \mu_{*} \mathscr{O}_{U_{g}}(1)$ is locally free and commutes with any base change.

\subsection{Grothendieck Embedding of the Relative Quot Scheme}

Let us fix a natural number $k \in \mathbb{N}$ and denote by $F$ the vector space $\mathbb{C}^{P(k)}$. Let $\mathrm{Q}_{g}(\mu, k, P) \subset$ Quot $_{F \otimes \mathscr{O}_{U_{g}}(-k) / U_{g} / \mathrm{H}_{g}}^{P}$ be the subscheme parametrizing quotients of pure dimension one. The forgetful functor gives a proper morphism $\pi: \mathrm{Q}_{g}(\mu, k, P) \rightarrow \mathrm{H}_{g}$ and the fibered product



is equipped with a universal quotient, flat over $\mathrm{Q}_{g}(\mu, k, P)$ (see [9])

$$
q_{Q_{g}}: F \otimes \phi^{*} \mathscr{O}_{U_{g}}(-k) \rightarrow \mathscr{F} \rightarrow 0 .
$$

There exists an integer $l_{1}$, such that, for all $l>l_{1}(l>k)$, there is an immersion $\pi \times i_{l}: \mathrm{Q}_{g}(\mu, k, P) \hookrightarrow \mathrm{H}_{g} \times \operatorname{Grass}\left(P(l), F \otimes H^{0}\left(\mathbf{P}^{M}, \mathcal{O}_{\mathbf{P}^{M}}(l-k)\right)\right)$. Composing $\pi \times i_{l}$ with the Plücker map, we get an immersion, that we denote in the same way 


$$
\pi \times i_{l}: \mathrm{Q}_{g}(\mu, k, P) \hookrightarrow \mathrm{H}_{g} \times \mathbf{P}\left(H_{2}\right),
$$

where $H_{2}:=\bigwedge^{P(l)}\left(F \otimes H^{0}\left(\mathbf{P}^{M}, \mathscr{O}_{\mathbf{P}^{M}}(l-k)\right)\right)$.

\subsection{Projective Embedding of Swamps Data}

By Theorem 3.9, there exists $N \in \mathbb{N}$ with the following property: given a stable curve of genus $g$ (with polarization $\mathscr{O}_{X}(1):=\omega_{X}^{\otimes 10}$ ), any $\delta$-semistable swamp $(\mathscr{F}, \phi)$ of type $\left(a, b, \mathscr{O}_{X}\right)$ with Hilbert polynomial $P$ defines a point in the projective bundle $\bar{\pi}: \mathbf{P}\left(\left(\left(F^{\otimes a}\right)^{\oplus b}\right)^{\vee} \otimes \mu_{*} \mathscr{O}_{U_{g}}(a k)\right) \rightarrow \mathrm{H}_{g}$ for any $k \geq N$ (recall that $\left.F=\mathbb{C}^{P(k)}\right)$. Since $\mu_{*} \mathscr{O}_{U_{g}}(a k)=p r_{1 *} \psi_{*}\left(\psi^{*} p r_{2}^{*} \mathscr{O}_{\mathbf{P}^{M}}(a k)\right)$, the natural surjection $p r_{2}^{*} \mathscr{O}_{\mathbf{P}^{M}}(a k) \rightarrow \psi_{*} \psi^{*} p r_{2}^{*} \mathscr{O}_{\mathbf{P}^{M}}(a k) \rightarrow 0$, induces a morphism

$$
\mathscr{O}_{H_{g}} \otimes_{\mathbb{C}} H^{0}\left(\mathbf{P}^{M}, \mathscr{O}_{\mathbf{P}^{M}}(a k)\right) \simeq p r_{1 *} p r_{2}^{*} \mathscr{O}_{\mathbf{P}^{M}}(a k) \longrightarrow \mu_{*} \mathscr{O}_{U_{g}}(a k),
$$

which will be denoted by $v_{k}^{\prime}$. By [10, Théorème 2.2.1. (ii)], there is a natural number $N^{\prime} \in \mathbb{N}$ that we can take greater than $N$, such that if $k \geq N^{\prime}$, then $v_{k}$ is surjective. Hence, we have a closed immersion of $\mathrm{H}_{g}$-schemes

$$
\mathbf{P}\left(\left(\left(F^{\otimes a}\right)^{\oplus b}\right)^{\vee} \otimes \mu_{*} \mathscr{O}_{U_{g}}(a k)\right) \longleftrightarrow \mathrm{H}_{g} \times \mathbf{P}\left(H_{3}\right),
$$

if $k \geq N^{\prime}$, where $\left.H_{3}=\left(\left(F^{\otimes a}\right)^{\oplus b}\right)^{\vee}\right) \otimes H^{0}\left(\mathbf{P}^{M}, \mathscr{O}_{\mathbf{P}^{M}}(a k)\right)$.

\subsection{Universal Parameter Space for Swamps}

Let $k$ be a natural number with $k \geq N^{\prime}$. Let

$$
Y:=\mathrm{Q}_{g}(\mu, k, P) \times_{H_{g}} \mathbf{P}\left(\left(\left(F^{\otimes a}\right)^{\oplus b}\right)^{\vee} \otimes \mu_{*} \mathscr{O}_{U_{g}}(a k)\right)
$$

be the pullback of $\bar{\pi}: \mathbf{P}\left(\left(\left(F^{\otimes a}\right)^{\oplus b}\right)^{\vee} \otimes \mu_{*} \mathscr{O}_{U_{g}}(a k)\right) \rightarrow H_{g}$ by $\pi: Q_{g}(\mu, k, P) \rightarrow$ $H_{g}$. We denote by $\pi_{1}$ and $\pi_{2}$ the projections of $Y$ onto the first and the second factor, respectively. Let $w: Y \rightarrow H_{g}$ be the induced projection. Giving $\pi_{2}$ is the same as giving a non-zero morphism

$$
\varphi_{Y}^{\prime}:\left(\left(F^{\otimes a}\right)^{\oplus b}\right) \otimes \mathscr{O}_{Y} \rightarrow w^{*} \mu_{*} \mathscr{O}_{U_{g}}(a k) \otimes \mathscr{L},
$$

while giving $\pi_{1}$ is the same as giving a quotient sheaf

$$
q_{Y}: F \otimes \mathscr{O}_{Y \times_{H_{g}} U_{g}} \rightarrow \mathscr{E}(k) \rightarrow 0
$$

on $Y \times_{H_{g}} U_{g}$. Let $\pi_{Y}$ and $\pi_{U_{g}}$ be the projections of $Y \times_{H_{g}} U_{g}$ onto $Y$ and $U_{g}$, respectively. Now, we can pull (13) back to $Y \times_{H_{g}} U_{g}$, and we get

$$
\varphi_{Y}^{\prime \prime}:\left(\left(F^{\otimes a}\right)^{\oplus b}\right) \otimes \mathscr{O}_{Y \times_{H_{g}} U_{g}} \rightarrow \pi_{Y}^{*} w^{*} \mu_{*} \mathscr{O}_{U_{g}}(a k) \otimes \pi_{Y}^{*} \mathscr{L} \rightarrow 0 .
$$

Note that $\pi_{Y}^{*} w^{*} \mu_{*} \mathscr{O}_{U_{g}}(a k) \otimes \pi_{Y}^{*} \mathscr{L}=\pi_{U_{g}}^{*} \mu^{*} \mu_{*} \mathscr{O}_{U_{g}}(a k) \otimes \pi_{Y}^{*} \mathscr{L}$. Composing $\varphi_{Y}^{\prime \prime}$ with the surjection $\pi_{U_{g}}^{*} \mu^{*} \mu_{*} \mathscr{O}_{U_{g}}(a k) \otimes \pi_{Y}^{*} \mathscr{L} \rightarrow \pi_{U_{g}}^{*} \mathscr{O}_{U_{g}}(a k) \otimes \pi_{Y}^{*} \mathscr{L} \rightarrow 0$, we get a morphism $\overline{\varphi_{Y}}:\left(\left(F^{\otimes a}\right)^{\oplus b}\right) \otimes \mathscr{O}_{Y \times_{H_{g}} U_{g}} \rightarrow \pi_{U_{g}}^{*} \mathscr{O}_{U_{g}}(a k) \otimes \pi_{Y}^{*} \mathscr{L}$. Let $\mathscr{K}$ be the kernel of $\left(q_{Y}^{\otimes a}\right)^{\oplus b}$ and denote by $\overline{\overline{\varphi_{Y}}}: \mathscr{K} \rightarrow \pi_{U_{g}}^{*} \mathscr{O}_{U_{g}}(a k) \otimes \pi_{Y}^{*} \mathscr{L}$ the restriction of $\overline{\varphi_{Y}}$ to $\mathscr{K}$. Since $\pi_{U_{g}}^{*} \mathscr{O}_{U_{g}}(a k) \otimes \pi_{Y}^{*} \mathscr{L}$ is flat over $Y$, there exists a closed subscheme $\mathcal{Z} \subset Y$ characterized by the fact that $\left.\overline{\overline{\varphi_{Y}}}\right|_{\mathcal{Z}}=0$. The restriction of $\overline{\varphi_{Y}}$ to $\mathcal{Z}$ lifts to $\left(\mathscr{E}(k)^{\otimes a}\right)^{\oplus b}$; that is, it factorizes through a morphism $\varphi_{\mathcal{Z}}:\left(\left.\mathscr{E}(k)\right|_{\mathcal{Z}} ^{\otimes a}\right)^{\oplus b} \rightarrow \pi_{U_{g}}^{*} \mathscr{O}_{U_{g}}(a k) \otimes \pi_{\mathcal{Z}}^{*}\left(\left.\mathscr{L}\right|_{\mathcal{Z}}\right)$, where $\pi_{\mathcal{Z}}$ is the projection of $\mathcal{Z} \times{ }_{H_{g}} U_{g}$ onto $\mathcal{Z},\left.\mathscr{E}(k)\right|_{\mathcal{Z}}$ is the restriction of $\mathscr{E}(k)$ to $\mathcal{Z} \times{ }_{H_{g}} U_{g}$, 
and $\left.\mathscr{L}\right|_{\mathcal{Z}}$ is the restriction of $\mathscr{L}$ to $\mathcal{Z}$. Then, the closed subscheme $\mathcal{Z} \subset Y$ carries a universal family of swamps over $\mathrm{H}_{g}$

$$
\begin{aligned}
& q_{\mathcal{Z}}:\left.F \otimes \mathscr{O}_{\mathcal{Z} \times_{H_{g}} U_{g}}(-k) \rightarrow \mathscr{E}\right|_{\mathcal{Z}}, \\
& \varphi_{\mathcal{Z}}:\left(\left.\mathscr{E}(k)\right|_{\mathcal{Z}} ^{\otimes a}\right)^{\oplus b} \rightarrow \pi_{U_{g}}^{*} \mathscr{O}_{U_{g}}(a k) \otimes \pi_{\mathcal{Z}}^{*}\left(\left.\mathscr{L}\right|_{\mathcal{Z}}\right) .
\end{aligned}
$$

\subsection{The Polarization of the Universal Parameter Space}

The action of $\mathrm{SL}_{n}$ on $F$ induces, naturally, actions on both spaces, $\mathrm{Q}_{g}(\mu, k, P)$ and $\mathbf{P}\left(\left(\left(F^{\otimes a}\right)^{\oplus b}\right)^{\vee} \otimes \mu_{*} \mathscr{O}_{U_{g}}(a k)\right)$. Likewise, the action of $\mathrm{SL}_{M+1}$ on $\mathrm{H}_{g}$ induces an action on them. The induced action on the first space is described in [17, Section 1.4], and the action on the second space can be described in the same terms. Thus, the group $\mathrm{SL}_{M+1} \times \mathrm{SL}_{n}$ acts on $Y$. On the other hand, we may choose $k, l$, and $s$ large enough (see Sects. 4.1, 4.2 and 4.3) to get an immersion

$$
j_{s, l, k}: Y \hookrightarrow \mathbf{P}\left(H_{1}\right) \times \mathbf{P}\left(H_{2}\right) \times \mathbf{P}\left(H_{3}\right),
$$

which is $\left(\mathrm{SL}_{M+1} \times \mathrm{SL}_{n}\right)$-equivariant. For each $i=1,2,3$, we denote by $\pi_{i}$ the projection of $\mathbf{P}\left(H_{1}\right) \times \mathbf{P}\left(H_{2}\right) \times \mathbf{P}\left(H_{3}\right)$ onto $\mathbf{P}\left(H_{i}\right)$. Given natural numbers, $\beta$ and $\gamma$, there are isomorphisms

$$
H^{0}\left(\mathbf{P}\left(H_{2}\right), \mathscr{O}_{\mathbf{P}\left(H_{2}\right)}(\beta)\right) \simeq S^{\beta} H_{2}, H^{0}\left(\mathbf{P}\left(H_{3}\right), \mathscr{O}_{\mathbf{P}\left(H_{3}\right)}(\gamma)\right) \simeq S^{\gamma} H_{3} .
$$

The Segre embedding, sg : $\mathbf{P}\left(H_{2}\right) \times \mathbf{P}\left(H_{3}\right) \rightarrow \mathbf{P}\left(S^{\beta} H_{2} \otimes S^{\gamma} H_{3}\right)$, is a closed immersion, and is determined by a surjection

$$
\left(S^{\beta} H_{2} \otimes S^{\gamma} H_{3}\right) \otimes \mathscr{O}_{\mathbf{P}\left(H_{2}\right) \times \mathbf{P}\left(H_{3}\right)} \rightarrow \pi_{2}^{*} \mathscr{O}_{\mathbf{P}\left(H_{2}\right)}(\beta) \otimes \pi_{3}^{*} \mathscr{O}_{\mathbf{P}\left(H_{3}\right)}(\gamma) \rightarrow 0 .
$$

Let us denote by $J$ the vector space $S^{\beta} H_{2} \otimes S^{\gamma} H_{3}$. Then, we have an isomorphism $s g^{*} \mathscr{O}_{\mathbf{P}(J)}(1) \simeq \pi_{2}^{*} \mathscr{O}_{\mathbf{P}\left(H_{2}\right)}(\beta) \otimes \pi_{3}^{*} \mathscr{O}_{\mathbf{P}\left(H_{3}\right)}(\gamma)$. Let us consider now the map

$$
\pi_{1} \times s g: \mathbf{P}\left(H_{1}\right) \times \mathbf{P}\left(H_{2}\right) \times \mathbf{P}\left(H_{3}\right) \hookrightarrow \mathbf{P}\left(H_{1}\right) \times \mathbf{P}(J) .
$$

For the polarization $\mathscr{O}(\alpha, 1)$ on $\mathbf{P}\left(H_{1}\right) \times \mathbf{P}(J)$, with $\alpha \in \mathbb{N}$, we have

$$
\left(\pi_{1} \times s g\right)^{*} \mathscr{O}(\alpha, 1)=\pi_{1}^{*} \mathscr{O}_{\mathbf{P}\left(H_{1}\right)}(\alpha) \otimes \pi_{2}^{*} \mathscr{O}_{\mathbf{P}\left(H_{2}\right)}(\beta) \otimes \pi_{3}^{*} \mathscr{O}_{\mathbf{P}\left(H_{3}\right)}(\gamma)
$$

From now onwards, $\beta$ and $\gamma$ are assumed to satisfy

$$
\frac{\beta}{\gamma}=\frac{P(l)-P(k)}{P(k)-a \delta} \delta,
$$

as in the fiber-wise problem (10).

\subsection{Proof of Theorem 4.1}

The action of $\mathrm{SL}_{M+1}$ on $Y$ induces a representation, $\xi_{i}: \mathrm{SL}_{M+1} \rightarrow \mathrm{SL}\left(H_{i}\right)$, for each $i=1,2,3$. Furthermore, $\xi_{2}$ and $\xi_{3}$ induce a representation $\omega$ : $\mathrm{SL}_{M+1} \rightarrow \mathrm{SL}(J)$. We will denote just by $\xi$ the representation $\xi_{1}$ and by $\rho_{H_{1}}: \mathbf{P}\left(H_{1}\right) \times \mathbf{P}(J) \rightarrow \mathbf{P}\left(H_{1}\right)$ the projection onto $\mathbf{P}\left(H_{1}\right)$. Recall that $\pi_{1}$ denotes the projection of $\mathbf{P}\left(H_{1}\right) \times \mathbf{P}\left(H_{2}\right) \times \mathbf{P}\left(H_{3}\right)$ onto $\mathbf{P}\left(H_{1}\right)$. From now onwards, the superscripts $\left\{s^{\prime}, s s^{\prime}\right\},\left\{s^{\prime \prime}, s s^{\prime \prime}\right\}$, and $\{s, s s\}$ will denote stability (semistability) with respect to the action of $\mathrm{SL}_{M+1}, \mathrm{SL}_{n}$ and $\mathrm{SL}_{M+1} \times \mathrm{SL}_{n}$, respectively, while the subscripts $[\cdot, \cdot]$ (or $[\cdot, \cdot, \cdot])$ will denote the polarization respect which we analyze the semistability condition. 
4.6.1. Step 1: Existence of the Quotient. Our aim is to show that $j_{s, l, k}(\mathcal{Z})$ is closed in $\left(\mathbf{P}\left(H_{1}\right) \times \mathbf{P}\left(H_{2}\right) \times \mathbf{P}\left(H_{3}\right)\right)_{[\alpha, \beta, \gamma]}^{s s}$.

Lemma 4.2 [17, Proposition 7.1.1]. There exists an integer $\alpha_{0}=\alpha_{0}(\xi, \omega)$, such that $\forall \alpha>\alpha_{0}, \rho_{H_{1}}^{-1}\left(\mathbf{P}\left(H_{1}\right)^{s^{\prime}}\right) \subset\left(\mathbf{P}\left(H_{1}\right) \times \mathbf{P}(J)\right)_{[\alpha, 1]}^{s^{\prime}}$.

Lemma 4.3 [17, Proposition 7.1.2]. There exists an integer $\alpha_{1}=\alpha_{1}(\xi, \omega)$, such that $\forall \alpha>\alpha_{1},\left(\mathbf{P}\left(H_{1}\right) \times \mathbf{P}(J)\right)_{[\alpha, 1]}^{s s^{\prime}} \subset \rho_{H_{1}}^{-1}\left(\mathbf{P}\left(H_{1}\right)^{s s^{\prime}}\right)$.

Proposition 4.4. There exists a natural number $\alpha \in \mathbb{N}$, such that (1) $j_{s, l, k}(\mathcal{Z})$ lies inside $\left(\mathbf{P}\left(H_{1}\right) \times \mathbf{P}\left(H_{2}\right) \times \mathbf{P}\left(H_{3}\right)\right)_{[\alpha, \beta, \gamma]}^{s^{\prime}}$ and (2) $j_{s, l, k}(\mathcal{Z})$ is closed inside $\left(\mathbf{P}\left(H_{1}\right) \times \mathbf{P}\left(H_{2}\right) \times \mathbf{P}\left(H_{3}\right)\right)_{[\alpha, \beta, \gamma]}^{s s^{\prime}}$.

Remark 4.5. Since $j_{s, l, k}$ is an immersion, we can identify $\mathcal{Z}$ with a subscheme of $\mathbf{P}\left(H_{1}\right) \times \mathbf{P}\left(H_{2}\right) \times \mathbf{P}\left(H_{3}\right)$.

Proof. Due to the previous lemmas, if $\alpha>\max \left\{\alpha_{0}, \alpha_{1}\right\}$, then

$$
\begin{aligned}
\rho_{H_{1}}^{-1}\left(\mathbf{P}\left(H_{1}\right)^{s^{\prime}}\right) & \subset\left(\mathbf{P}\left(H_{1}\right) \times \mathbf{P}(J)\right)_{[\alpha, 1]}^{s^{\prime}}, \\
\left(\mathbf{P}\left(H_{1}\right) \times \mathbf{P}(J)\right)_{[\alpha, 1]}^{s s^{\prime}} & \subset \rho_{H_{1}}^{-1}\left(\mathbf{P}\left(H_{1}\right)^{s s^{\prime}}\right) .
\end{aligned}
$$

If we apply $(i d \times s g)^{-1}$ to (18) and (19), we find the relations

$$
\begin{aligned}
& \pi_{1}^{-1}\left(\mathbf{P}\left(H_{1}\right)^{s^{\prime}}\right)=(i d \times s g)^{-1} \rho_{H_{1}}^{-1}\left(\mathbf{P}\left(H_{1}\right)^{s^{\prime}}\right), \\
& (i d \times s g)^{-1} \rho_{H_{1}}^{-1}\left(\mathbf{P}\left(H_{1}\right)^{s^{\prime}}\right) \subset(i d \times s g)^{-1}\left(\mathbf{P}\left(H_{1}\right) \times \mathbf{P}(J)\right)_{[\alpha, 1]}^{s^{\prime},}, \\
& (i d \times s g)^{-1}\left(\mathbf{P}\left(H_{1}\right) \times \mathbf{P}(J)\right)_{[\alpha, 1]}^{s^{\prime}}=\left(\mathbf{P}\left(H_{1}\right) \times \mathbf{P}\left(H_{2}\right) \times \mathbf{P}\left(H_{3}\right)\right)_{[\alpha, \beta, \gamma]}^{s^{\prime}}, \\
& \left(\mathbf{P}\left(H_{1}\right) \times \mathbf{P}\left(H_{2}\right) \times \mathbf{P}\left(H_{3}\right)\right)_{[\alpha, \beta, \gamma]}^{s s^{\prime}}=(i d \times s g)^{-1}\left(\mathbf{P}\left(H_{1}\right) \times \mathbf{P}(J)\right)_{[\alpha, 1]}^{s s^{\prime},}, \\
& (i d \times s g)^{-1}\left(\mathbf{P}\left(H_{1}\right) \times \mathbf{P}(J)\right)_{[\alpha, 1]}^{s s^{\prime}} \subset(i d \times s g)^{-1} \rho_{H_{1}}^{-1}\left(\mathbf{P}\left(H_{1}\right)^{s s^{\prime}}\right), \\
& (i d \times s g)^{-1} \rho_{H_{1}}^{-1}\left(\mathbf{P}\left(H_{1}\right)^{s s^{\prime}}\right)=\pi_{1}^{-1}\left(\mathbf{P}\left(H_{1}\right)^{s s^{\prime}}\right) .
\end{aligned}
$$

(1) Since $H_{g} \subset \mathbf{P}\left(H_{1}\right)^{s^{\prime}}$ and (20), (21), (22) hold, we have an inclusion

$$
\mathcal{Z} \subset H_{g} \times \mathbf{P}\left(H_{2}\right) \times \mathbf{P}\left(H_{3}\right) \subset\left(\mathbf{P}\left(H_{1}\right) \times \mathbf{P}\left(H_{2}\right) \times \mathbf{P}\left(H_{3}\right)\right)_{[\alpha, \beta, \gamma]}^{s^{\prime}} .
$$

(2) $\mathrm{H}_{g}$ is closed in $\mathbf{P}\left(H_{1}\right)^{s s^{\prime}}$ (see Sect. 4.1), so $\mathrm{H}_{g} \times \mathbf{P}\left(H_{2}\right) \times \mathbf{P}\left(H_{3}\right)$ is closed in $\rho_{H_{1}}^{-1}\left(\mathbf{P}\left(H_{1}\right)^{s s^{\prime}}\right)$. Also, $\mathcal{Z}$ is closed in $H_{g} \times \mathbf{P}\left(H_{1}\right) \times \mathbf{P}\left(H_{3}\right)$ because it is proper over $\mathrm{H}_{g}$. Thus, it is so also as a subscheme of $\pi_{1}^{-1}\left(\mathbf{P}\left(H_{1}\right)^{s s^{\prime}}\right)$. The relations (23), (24), (25), (26) yield

$$
\mathcal{Z} \subset\left(\mathbf{P}\left(H_{1}\right) \times \mathbf{P}\left(H_{2}\right) \times \mathbf{P}\left(H_{3}\right)\right)_{[\alpha, \beta, \gamma]}^{s s^{\prime}} \subset \pi_{1}^{-1}\left(\mathbf{P}\left(H_{1}\right)^{s s^{\prime}}\right) .
$$

Therefore, $\mathcal{Z}$ is closed in $\left(\mathbf{P}\left(H_{1}\right) \times \mathbf{P}\left(H_{2}\right) \times \mathbf{P}\left(H_{3}\right)\right)_{[\alpha, \beta, \gamma]}^{s s^{\prime}}$.

Let us define $\mathcal{Z}_{[\alpha, \beta, \gamma]}^{s s}:=\mathcal{Z} \cap\left(\mathbf{P}\left(H_{1}\right) \times \mathbf{P}\left(H_{2}\right) \times \mathbf{P}\left(H_{3}\right)\right)_{[\alpha, \beta, \gamma]}^{s s}$. Since $\left(\mathbf{P}\left(H_{1}\right) \times \mathbf{P}\left(H_{2}\right) \times \mathbf{P}\left(H_{3}\right)\right)_{[\alpha, \beta, \gamma]}^{s s}$ lies inside $\left(\mathbf{P}\left(H_{1}\right) \times \mathbf{P}\left(H_{2}\right) \times \mathbf{P}\left(H_{3}\right)\right)_{[\alpha, \beta, \gamma]}^{s s^{\prime}}$, 
it follows that $\mathcal{Z}_{[\alpha, \beta, \gamma]}^{s s}$ is closed in $\left(\mathbf{P}\left(H_{1}\right) \times \mathbf{P}\left(H_{2}\right) \times \mathbf{P}\left(H_{3}\right)\right)_{[\alpha, \beta, \gamma]}^{s s}$. Therefore

$$
\mathcal{T}_{P, g, a, b}^{\delta-(\mathrm{s}) \mathrm{s}}:=\mathcal{Z}_{[\alpha, \beta, \gamma]}^{s(s)} /\left(\mathrm{SL}_{M+1} \times \mathrm{SL}_{n}\right)
$$

exists and is projective, and there is a well-defined map $\Theta_{s w}: \mathcal{T}_{P, g, a, b}^{\delta-(s) s} \rightarrow \overline{\mathrm{M}}_{g}$, such that $\Theta_{s w}^{-1}([X])=\mathcal{T}_{P, \mathscr{O}_{X}, a, b}^{\delta-(\mathrm{s}) \mathrm{s}}(X) / \operatorname{Aut}(X)$ for any stable curve $[X] \in \overline{\mathrm{M}}_{g}$.

\subsubsection{Step 2: Closed Points of the Quotient.}

Proposition 4.6. There is a one-to-one correspondence between closed points of $\mathcal{Z}_{[\alpha, \beta, \gamma]}^{s(s)}$ and pairs $(X,(q, \phi))$, where $X$ is a 10-canonical stable curve of genus $g, q: F \otimes \mathscr{O}_{X}(-k) \rightarrow \mathscr{F} \rightarrow 0$ is a quotient of uniform rank $r$, such that $H^{0}(q(k)): F \rightarrow H^{0}(X, \mathscr{F}(k))$ is an isomorphism, and $(\mathscr{F}, \phi)$ is a $\delta$ (semi)stable swamp of type $\left(a, b, \mathscr{O}_{X}\right)$ with Hilbert polynomial $P$.

Proof. Note that we have the inclusion $\mathcal{Z}_{[\alpha, \beta, \gamma]}^{s(s)} \subset \mathcal{Z}_{[\alpha, \beta, \gamma]}^{s^{\prime \prime}\left(s^{\prime \prime}\right)}$. If we prove the equality $\mathcal{Z}_{[\alpha, \beta, \gamma]}^{s(s)}=\mathcal{Z}_{[\alpha, \beta, \gamma]}^{s^{\prime \prime}\left(s^{\prime \prime}\right)}$, then the result will follow from Theorem 3.11 and the construction of $\mathcal{Z}_{[\alpha, \beta, \gamma]}^{s s}$. We use the Hilbert-Mumford criterium to prove it. Let us consider the representations

$$
\begin{aligned}
& \xi^{\alpha}: \mathrm{SL}_{M+1} \times \mathrm{SL}_{n} \rightarrow \mathrm{SL}_{M+1} \rightarrow \mathrm{SL}\left(S^{\alpha}\left(H_{1}\right)\right), \\
& \omega_{1}: \mathrm{SL}_{M+1} \times \mathrm{SL}_{n} \rightarrow \mathrm{SL}\left(S^{\beta}\left(H_{2}\right)\right), \\
& \omega_{2}: \mathrm{SL}_{M+1} \times \mathrm{SL}_{n} \rightarrow \mathrm{SL}\left(S^{\gamma}\left(H_{3}\right)\right) .
\end{aligned}
$$

The representations $\omega_{1}$ and $\omega_{2}$ give us a representation $\omega=\omega_{1} \otimes \omega_{2}: \mathrm{SL}_{M+1} \times$ $\mathrm{SL}_{n} \rightarrow \mathrm{SL}(J)$. Let $\eta \in \mathcal{Z}_{[\alpha, \beta, \gamma]}^{s^{\prime \prime}\left(s^{\prime \prime}\right)}$ be a closed point and let us define $\left(\eta_{1}, \eta_{2}\right):=$ $\left(\left(\pi_{1} \times s g\right) \circ j_{s, l, k}\right)(\eta)$. Let $\lambda: \mathbb{G}_{m} \rightarrow \mathrm{SL}_{M+1} \times \mathrm{SL}_{n}$ be a non-trivial 1-PS given by $\lambda_{1}: \mathbb{G}_{m} \rightarrow \mathrm{SL}_{M+1}$ and $\lambda_{2}: \mathbb{G}_{m} \rightarrow \mathrm{SL}_{n}$. Now, the equality is proved through the same argument as the one given in [17, Proposition 8.2.1].

\subsubsection{Step 3: The Universal Natural Transformation.}

Proposition 4.7. There exists a universal natural transformation

$$
\Psi: \operatorname{mathbfS} w_{P, g, a, b}^{\delta-(s) s} \longrightarrow \operatorname{Hom}\left(-, \mathcal{T}_{P, g, a, b}^{\delta-(s) s}\right) .
$$

Proof. Let $T$ be a scheme and $\eta \in \mathbf{S w}_{P, g, a, b}^{\delta \text {-(s)s }}(T)$ a $T$-point, which consists of a tuple $\left(\pi, \mathscr{F}_{T}, \mathscr{N}, \phi\right)$, where $\pi: X_{T} \rightarrow T$ is a flat family of stable curves of genus $g$ with relative polarization $\mathscr{O}_{X_{T}}(1):=\omega_{X_{T} / T}^{\otimes 10}, \mathscr{F}_{T}$ is a flat family of coherent sheaves of pure dimension one with Hilbert polynomial $P$ and uniform rank $r, \mathscr{N}$ is an invertible sheaf on $T$, and $\phi:\left(\mathscr{F}_{T}^{\otimes a}\right)^{\oplus b} \rightarrow \pi^{*} \mathscr{N}$ is a $\delta$-(semi)stable swamp. Let $k \geq N^{\prime}$ be the natural number fixed for the construction of $\mathcal{T}_{P, g, a, b}^{\delta \text {-(s)s }}$ (see Sect. 4.3). Then, $R^{1} \pi_{*} \mathscr{F}_{T}(k)=0$ and $\pi_{*} \mathscr{F}_{T}(k)$ is locally free of rank $n=P(k)$. On the other hand, $R^{1} \pi_{*} \mathscr{O}_{X_{T}}(1)=0$ and $\pi_{*} \mathscr{O}_{X_{T}}(1)$ is locally free of rank $N+1=10(2 g-2)-g+1$. Let $\left\{U_{i}\right\}$ be an open cover of $T$, such that $\left.\left(\pi_{*} \mathscr{F}_{T}(k)\right)\right|_{U_{i}} \simeq F \otimes \mathscr{O}_{U_{i}}$ and $\left.\left(\pi_{*} \mathscr{O}_{X_{T}}(1)\right)\right|_{U_{i}} \simeq$ $\mathbb{C}^{M+1} \otimes \mathscr{O}_{U_{i}}$, where $F=\mathbb{C}^{n}$. This trivializations induce surjections

$$
q_{2, W_{i}}:\left.\left.\mathbb{C}^{M+1} \otimes \mathscr{O}_{X_{T}}\right|_{W_{i}} \rightarrow \mathscr{O}_{X_{T}}(1)\right|_{W_{i}} \rightarrow 0,
$$




$$
q_{1, W_{i}}:\left.\left.F \otimes \mathscr{O}_{X_{T}}(-k)\right|_{W_{i}} \rightarrow \mathscr{F}_{T}\right|_{W_{i}} \rightarrow 0,
$$

where $W_{i}=\pi^{-1}\left(U_{i}\right)$. Composing $\left.\phi(a k)\right|_{W_{i}}$ with $\left(q_{1, W_{i}}(k)^{\otimes a}\right)^{\oplus b}$ and taking the pushforward by $\pi$, we get a morphism

$$
\phi_{i}:\left.\left.\left(F^{\otimes a}\right)^{\oplus b} \otimes \mathscr{O}_{T}\right|_{U_{i}} \rightarrow\left(\left.\mathscr{N}\right|_{U_{i}}\right) \otimes \pi_{*} \mathscr{O}_{X_{T}}(a k)\right|_{U_{i}}
$$

The first surjection, (27), gives an embedding $q_{2, W_{i}}: W_{i} \hookrightarrow U_{i} \times \mathbf{P}^{M}$, while the second surjection, (28), defines a map $q_{1, W_{i}}: U_{i} \rightarrow \mathrm{Q}_{g}(\mu, k, P)$. Finally, the morphism $\phi_{i}$ defines a map $\phi_{i}: U_{i} \rightarrow \mathbf{P}\left(\left(\left(F^{\otimes a}\right)^{\oplus b}\right)^{\vee} \otimes \mu_{*} \mathscr{O}_{U_{g}}(a k)\right)$ as well. Therefore, $q_{1, W_{i}}$ and $\phi_{i}$ define a map $\psi_{i}: U_{i} \rightarrow Y$. Consider two open subsets $U_{i}, U_{j}$, and let $U_{i j}=U_{i} \cap U_{j}$ be the intersection. Then, $\phi_{i}$ and $\phi_{j}$ define maps $U_{i j} \rightarrow Y$ which differ by a $U_{i j}$-valued point of the group scheme $\mathrm{SL}_{n} \times \mathrm{SL}_{M+1}$. Furthermore, $\operatorname{Im}\left(U_{i j} \rightarrow Y\right)$ lies inside $\mathcal{Z}_{[\alpha, \beta, \gamma]}^{s s}$, because $\eta$ is $\delta$-semistable. Therefore, there is a well-defined morphism $T \rightarrow \mathcal{T}_{P, g, a, b}^{\delta \text {-(s)s }}$. This shows the existence of a natural transformation

$$
\Psi: \mathbf{S w}_{P, g, a, b}^{\delta-(\mathrm{s}) \mathrm{s}} \longrightarrow \operatorname{Hom}\left(-, \mathcal{T}_{P, g, a, b}^{\delta-(\mathrm{s}) \mathrm{s}}\right) .
$$

Let $\mathcal{M}$ be a scheme and suppose that there exists a natural transformation $\Psi^{\prime}: \mathbf{S w}_{P, g, a, b}^{\delta-(\mathrm{s}) \mathrm{s}} \longrightarrow \operatorname{Hom}(-, \mathcal{M})$. There is a canonical $\phi_{\text {univ }} \in \mathbf{S w}_{P, g, a, b}^{\delta-(\mathrm{s}) \mathrm{s}}\left(\mathcal{Z}_{[\alpha, \beta, \gamma]}^{(\mathrm{s}) \mathrm{s}}\right)$ corresponding to the universal family (see (15)). The morphism $\Psi^{\prime}\left(\phi_{\text {univ }}\right)$ is $\mathrm{SL}_{n} \times \mathrm{SL}_{M+1}$-invariant, so it descends to a morphism $\mathcal{T}_{P, g, a, b}^{\delta \text {-(s)s }} \longrightarrow \mathcal{M}$, which defines a map $\Psi^{\prime \prime}: \operatorname{Hom}\left(-, \mathcal{T}_{P, g, a, b}^{\delta-(\mathrm{s}) \mathrm{s}}\right) \rightarrow \operatorname{Hom}(-, \mathcal{M})$. Clearly, $\Psi^{\prime \prime} \circ \Psi=\Psi^{\prime}$.

\section{A Compactification of the Universal Moduli Space of Principal $G$-bundles}

Let $G$ be a semisimple linear algebraic group and $\rho: G \hookrightarrow \operatorname{SL}(V)$ a faithful representation of dimension $r$. Let $g \geq 2$ be a natural number, $\delta \in \mathbb{Q}_{>0}$ a rational number, and $P$ a polynomial of degree one with integral coefficients.

\subsection{The Parameter Space}

Let $\mathrm{Q}_{g}(\mu, k, P)$ be the Quot scheme that was considered in the case of swamps. Let us consider the affine $\mathrm{H}_{g}$-scheme defined by

$$
\mathrm{H}(V, s, k):=\bigoplus_{i=1}^{s} \underline{\operatorname{Hom}}_{\mathrm{H}_{g}}\left(S^{i}\left(V \otimes \mathbb{C}^{n}\right) \otimes \mathscr{O}_{\mathrm{H}_{g}}, \mu_{*} \mathscr{O}_{U_{g}}(i k)\right) \stackrel{\kappa}{\longrightarrow} \mathrm{H}_{g} .
$$

By [14, Section 6.1] and the results of Sect. 3, every $\delta$-semistable singular principal $G$-bundle over a semistable curve of genus $g$ determines a point in $\mathrm{Q}_{g}(\mu, k, P) \times_{\mathrm{H}_{g}} \mathrm{H}(V, s, k)$ if $s$ and $k$ are large enough. Denote by $\bar{\kappa}$ and $\bar{\mu}$ the projections

$$
\begin{aligned}
& \left(\mathrm{Q}_{g}(\mu, k, P) \times \times_{\mathrm{H}_{g}} \mathrm{H}(V, s, k)\right) \times \times_{\mathrm{H}_{g}} U_{g} \longrightarrow U_{g}, \\
& \left(\mathrm{Q}_{g}(\mu, k, P) \times \times_{\mathrm{H}_{g}} \mathrm{H}(V, s, k)\right) \times \times_{\mathrm{H}_{g}} U_{g} \longrightarrow \mathrm{H}_{g},
\end{aligned}
$$

respectively. The goal now is to put a scheme structure on the locus given by the points $([q],[k]) \in \mathrm{Q}_{g}(\mu, k, P) \times_{\mathrm{H}_{g}} \mathrm{H}(V, s, k)$ that come from a morphism of algebras $S^{\bullet}(V \otimes \mathscr{F})^{G} \rightarrow \mathscr{O}_{X}$. For the sake of clarity, let us denote $\mathrm{E}_{g}:=$ 
$\left(\mathrm{Q}_{g}(\mu, k, P) \times_{\mathrm{H}_{g}} \mathrm{H}(V, s, k)\right) \times_{\mathrm{H}_{g}} U_{g}$. For each $i \in \mathbb{N}$, we consider the universal morphism

$$
\varphi^{\prime i}: S^{i}(V \otimes W) \otimes \mathscr{O}_{\mathrm{E}_{g}} \rightarrow \bar{\mu}^{*} \bar{\mu}_{*}\left(\bar{\kappa}^{*} \mathscr{O}_{U_{g}}(i k)\right)
$$

on $\mathrm{E}_{g}$. It leads to $S^{i}(V \otimes W) \otimes \mathscr{O}_{\mathrm{E}_{g}} \rightarrow \bar{\kappa}^{*} \mathscr{O}_{U_{g}}(i k)=\mathscr{O}_{\mathrm{E}_{g}}(i k)$ when composed with the evaluation map $\bar{\mu}^{*} \bar{\mu}_{*}\left(\bar{\kappa}^{*} \mathscr{O}_{U_{g}}(i k)\right) \rightarrow \bar{\kappa}^{*} \mathscr{O}_{U_{g}}(i k) \rightarrow 0$. Summing up these morphisms, we get $\varphi_{\mathrm{E}_{g}}: \mathscr{V}_{\mathrm{E}_{g}}:=\bigoplus_{i=1}^{s} S^{i}\left(V \otimes W \otimes \mathscr{O}_{\mathrm{E}_{g}}(-k)\right) \rightarrow \mathscr{O}_{\mathrm{E}_{g}}$. Let $\tau_{\mathrm{E}_{g}}^{\prime}: S^{\bullet}\left(\mathscr{V}_{\mathrm{E}_{g}}\right) \rightarrow \mathscr{O}_{\mathrm{E}_{g}}$ be the corresponding morphism of algebras. Consider the universal quotient (12), $q_{Q_{g}}: F \otimes \phi^{*} \mathscr{O}_{Q_{g} \times_{H_{g}} U_{g}}(-k) \rightarrow \mathscr{E} \rightarrow 0$, on $\mathrm{Q}_{g}(\mu, k, P) \times_{\mathrm{H}_{g}} U_{g}$. Pulling it back to $\mathrm{E}_{g}$, we get a quotient $q_{Q_{g} \times_{H_{g}} H}$ : $F \otimes \mathscr{O}_{\mathrm{E}_{g}}(-k) \rightarrow \overline{\mathscr{E}} \rightarrow 0$, and, therefore, a chain of surjections

$$
S^{\bullet} \mathscr{V}_{\mathrm{E}_{g}} \longrightarrow S^{\bullet}\left(V \otimes F \otimes \mathscr{O}_{\mathrm{E}_{g}}(-k)\right) \longrightarrow S^{\bullet}(V \otimes \overline{\mathscr{E}}) \longrightarrow S^{\bullet}(V \otimes \overline{\mathscr{E}})^{G}
$$

Then, there exists a closed subscheme $\mathrm{D}_{g} \subset \mathrm{Q}_{g}(\mu, k, P) \times{ }_{\mathrm{H}_{g}} \mathrm{H}(V, s, k)$ (follows as in [20, Section 5.1]) over which the morphism $\tau_{\mathrm{E}_{g}}^{\prime}: S^{\bullet} \mathscr{V}_{\mathrm{E}_{g}} \rightarrow \mathscr{O}_{\mathrm{E}_{g}}$ lifts to a morphism of algebras $\tau_{\mathrm{D}_{g}}: S^{\bullet}\left(V \otimes \overline{\mathscr{E}}_{\left.\right|_{\mathrm{D}_{g}}}\right)^{G} \rightarrow \mathscr{O}_{\mathrm{D}_{g}}$, where $\left.\overline{\mathscr{E}}\right|_{\mathrm{D}_{g}}$ is the restriction of $\overline{\mathscr{E}}$ to $\mathrm{D}_{g} \times_{H_{g}} U_{g} \subset \mathrm{E}_{g}$. This scheme carries a universal family of singular principal bundles, $\left(q_{\mathrm{D}_{g}}, \tau_{\mathrm{D}_{g}}\right)$.

\subsection{The Group Action and the Existence of the Quotient}

There are two groups acting on $\mathrm{D}_{g}$. The action of the group $\mathrm{SL}_{M+1}$ on $\mathrm{H}_{g}$ lifts to an action on $\mathrm{D}_{g}$, while the group $\mathrm{GL}_{n}$ is acting on both, $\mathrm{Q}_{g}(\mu, k, P)$ and $\mathrm{H}(V, s, k)$. The group $\mathrm{GL}_{n}$ leaves invariant $\mathrm{D}_{g}$, so $\mathrm{GL}_{n}$ is acting on $\mathrm{D}_{g}$ as well. Again, as in the fiber-wise problem, we can study the quotient by studying separately the actions of $\mathbb{C}^{*}$ and $\mathrm{SL}_{n}$ (see [20, Section 5.2]).

Let $\mathcal{Z}$ be the parameter space for swamps of the form $\phi:((V \otimes$ $\left.\mathscr{F})^{\otimes a}\right)^{\oplus b} \rightarrow \mathscr{O}_{X}$ (see Sect. 4.4). The injective map (4) shows that there is a well-defined morphism of $\mathrm{H}_{g}$-schemes Swamp : $\mathrm{D}_{g} \longrightarrow \mathcal{Z}$ which is $\mathbb{C}^{*}$ invariant and $\mathrm{SL}_{n}$-equivariant, injective and proper. Thus, it induces a morphism $\overline{\text { Swamp }}: \overline{\mathrm{D}_{g}}:=\mathrm{D}_{g} / / \mathbb{C}^{*} \longrightarrow \mathcal{Z}$ which is $\mathrm{SL}_{n}$-equivariant, injective and proper. Finally, by Definition 2.5 and Theorem 4.4 we conclude that

$$
\operatorname{SPB}(\rho)_{P, g}^{\delta-(\mathrm{s}) \mathrm{s}}:=\overline{\mathrm{D}_{g}} / /\left(\mathrm{SL}_{M+1} \times \mathrm{SL}_{n}\right)
$$

exists and is projective, and there is a well-defined map $\Theta_{s b}: \operatorname{SPB}(\rho)_{P, g}^{\delta \text {-(s)s }} \rightarrow$ $\overline{\mathrm{M}}_{g}$ satisfying that $\Theta_{s b}^{-1}([X])=\operatorname{SPB}(\rho)_{P, X}^{\delta \text {-(s) } \mathrm{s}} / \operatorname{Aut}(X)$ for any stable curve $[X] \in \overline{\mathrm{M}}_{g}$.

\subsection{Proof of Theorem 1.1}

Using the same arguments as those given in [20, Proposition 5.1, Proposition 5.2 , Theorem 5.3.], and the results of Sect. 4 , it follows that $\operatorname{SPB}(\rho)_{P, g}^{\delta-(\mathrm{s}) \mathrm{s}}$ is a projective coarse moduli space for the moduli functor

$$
\mathbf{S P B}_{P, g}^{\delta-(\mathrm{s}) \mathrm{s}}(\rho)(T)=\left\{\begin{array}{l}
\text { isomorphism classes of pairs }\left(X_{T},\left(\mathscr{F}_{T}, \tau_{T}, \mathscr{N}\right)\right), \\
\text { where } X_{T} \text { is a stable curve of genus } g \text { flat over } \\
T \text { and }\left(\mathscr{F}_{T}, \tau_{T}, \mathscr{N}\right) \text { is a family of } \delta \text {-(semi)stable } \\
\text { singular principal } G \text { bundles over } T \text { with Hilbert } \\
\text { polynomial } P \text { and type }\left(a, b, \mathscr{O}_{X_{T}}\right)
\end{array}\right\} .
$$


Let $r \in \mathbb{N}$ and $h=10(2 g-2)$. Assume now that $P(k)=(r h) k+r(1-g)$. This is the Hilbert polynomial of coherent sheaves of uniform rank $r$ and degree 0 over any stable curve $X$ with polarization $\omega_{X}^{\otimes 10}$. By [17, Lemma 8.1], there exists a closed, hence projective, subscheme $\operatorname{SPB}(\rho)_{r, g}^{\delta-(\mathrm{s}) \mathrm{s}}$ coarsely representing the sub-functor given by pairs $(X,(\mathscr{F}, \tau))$, where $\mathscr{F}$ has uniform rank $r$. The restriction of $\Theta_{s b}$ to $\operatorname{SPB}(\rho)_{r, g}^{\delta-(\mathrm{s}) \mathrm{s}}$ gives the desired map, which we denote by $\Theta_{b}$. By [21, Theorem 3.7], there exists $\delta_{\infty} \in \mathbb{Q}_{>0}$, depending only on $a, b, P$, such that the associated swamp of any $\delta$-semistable singular principal $G$-bundle on a smooth projective curve of genus $g,(\mathscr{F}, \tau)$, is generically semistable, and by [24, Corollary 4.1.2] and [25, Remark 2.3.4.4], this means that $(\mathscr{F}, \tau)$ is honest. Finally, [20, Paragraph 6.1] implies $\operatorname{SPB}(\rho)_{r, X}^{\delta-(\mathrm{s}) \mathrm{s}}=$ $\mathrm{M}_{X}(G)$ for any $\delta>\delta_{\infty}$ and any smooth projective curve of genus $g$. Therefore, if $\delta>\delta_{\infty}$, we have $\Theta_{b}^{-1}([X])=\mathrm{M}_{X}(G) / \operatorname{Aut}(X)$ for every smooth projective curve of genus $g$, so $\operatorname{SPB}(\rho)_{r, g}^{\delta-(\mathrm{s}) \mathrm{s}}$ is a compactification of the moduli problem defined by pairs $(X, \mathscr{P})$, where $X$ is a smooth projective curve of genus $g$ and $\mathscr{P}$ is a principal $G$-bundle.

\section{Acknowledgements}

Some results of this paper were obtained during the author's Ph.D. thesis period at Freie Universität Berlin. The author would like to thank Professor Alexander Schmitt for his encouragement and support.

Funding Open Access funding provided thanks to the CRUE-CSIC agreement with Springer Nature.

Open Access. This article is licensed under a Creative Commons Attribution 4.0 International License, which permits use, sharing, adaptation, distribution and reproduction in any medium or format, as long as you give appropriate credit to the original author(s) and the source, provide a link to the Creative Commons licence, and indicate if changes were made. The images or other third party material in this article are included in the article's Creative Commons licence, unless indicated otherwise in a credit line to the material. If material is not included in the article's Creative Commons licence and your intended use is not permitted by statutory regulation or exceeds the permitted use, you will need to obtain permission directly from the copyright holder. To view a copy of this licence, visit http:// creativecommons.org/licenses/by/4.0/.

Publisher's Note Springer Nature remains neutral with regard to jurisdictional claims in published maps and institutional affiliations.

\section{References}

[1] Balaji, V.: Torsors on semistable curves and degenerations. (2020). arXiv:1901.01529 [v4]

[2] Bhosle, U.: Tensor fields and singular principal bundles. Int. Math. Res. Not. 2004, 3057-3077 (2004) 
[3] Caporaso, L.: A compactification of the universal Picard variety over the moduli space of stable curve. J. Am. Math. Soc. 7, 589-660 (1994)

[4] Deligne, P., Mumford, D.: The irreducibility of the space of curves of given genus. Publications mathématiques de l'IHÉS 36, 75-109 (1969)

[5] Faltings, G.: Moduli stacks for bundles on semistable curves. Math. Ann. 304, 489-515 (1996)

[6] Gieseker, D.: Lectures on Moduli of Curves. Springer, Heidelberg (1982)

[7] Gómez, T., Sols, I.: Stable tensors and moduli space of orthogonal sheaves. (2001). arXiv: math.AG/0103150

[8] Grothendieck, A.: Sur la classification des fibres holomorphes sur la sphere de Riemann. Am. J. Math. 79, 121-138 (1957)

[9] Grothendieck, A.: Fondements de la géométrie algébrique [Extraits du Séminaire Bourbaki, 1957-1962]

[10] Grothendieck, A.: Éléments de géométrie algébrique : III. Étude cohomologique des faisceaux cohérents, Première partie, Publications Mathématiques de l'IHÉS, 11, 5-167 (1961)

[11] Langer, A.: Moduli spaces of principal bundles on singular varieties. Kyoto J. Math. 53, 3-23 (2013)

[12] Matsumura, H.: Commutative Ring Theory. Cambridge University Press, Cambridge (1986)

[13] Muñoz Castañeda, A.L.: Principal G-bundles on nodal curves. PhD thesis, Berlin (2017). ii+158 pp. Available at http://www.diss.fu-berlin.de/diss/ content/below/index.xml

[14] Muñoz Castañeda, A.L.: On the moduli spaces of singular principal bundles on stable curves. Adv. Geom. 20, 573-584 (2020)

[15] Mumford, D.: Projective invariants of projective structures and applications. In: Proceedings of the International Congress of Mathematicians, pp. 526-530 (1962)

[16] Mumford, D.: Red Book of Varieties and Schemes. Springer, Berlin (1999)

[17] Pandharipande, R.: A compactification over $\overline{\mathrm{M}} g$ of the universal moduli space of slope-semiestable vector bundles. J. Am. Math. Soc. 9, 425-471 (1996)

[18] Ramanathan, A.: Moduli of principal bundles over algebraic curves I. Proc. Indian Acad. Sci. 106, 301-328 (1996)

[19] Ramanathan, A.: Moduli of principal bundles over algebraic curves II. Proc. Indian Acad. Sci. 106, 421-449 (1996)

[20] Schmitt, A.: Singular principal bundles over higher-dimensional manifolds and their moduli spaces. Int. Math. Res. Not. 2002, 1183-1210 (2002)

[21] Schmitt, A.: Global boundedness for decorated sheaves. Int. Math. Res. Not. 68, 3637-3671 (2004)

[22] Schmitt, A.: The Hilbert compactification of the universal moduli space of semistable vector bundles over smooth curves. J. Differ. Geom. 66, 169-209 (2004)

[23] Schmitt, A.: Moduli spaces for semistable honest singular principal G-bundles on a nodal curve which are compatible with degenerations-A remark on Bhosle's paper Tensor fields and singular principal bundles". Int. Math. Res. Not. 2005, 1427-1436 (2005) 
[24] Schmitt, A.: Singular principal G-bundles on nodal curves. J. Eur. Math. Soc. 7, 215-251 (2005)

[25] Schmitt, A.: Geometric Invariant Theory and Decorated Principal Bundles. EMS Zurich Lectures in Advances Mathematics, Zurich (2008)

[26] Serre, J.P.: Espaces fibrés algébriques, Séminaire Claude Chevalley, Tome 3, Exposé (1), p. 37 (1958)

[27] Seshadri, C.S.: Space of unitary vector bundles on a compact Riemann surface. Ann. Math. 85, 303-336 (1967)

[28] Siedman, J.: On the Castelnuovo-Mumford regularity of products of ideal sheaves. Adv. Geom. 2, 219-229 (2002)

[29] Sun, X.: Degenerations of SL(r)-bundles on a reducible curve. Proceedings of the Symposium on Algebraic Geometry in East Asia, pp. 3-10 (2001)

[30] Sun, X.: Moduli spaces of SL(r)-bundles on singular irreducible curves. Asian J. Math. 7, 609-626 (2003)

\author{
Ángel Luis Muñoz Castañeda \\ Departamento de Matemáticas \\ Universidad de León \\ León \\ Spain \\ e-mail: amunc@unileon.es
}

Received: August 19, 2020.

Revised: April 13, 2021.

Accepted: December 6, 2021. 